\title{
Simultaneous Geologic Scenario Identification and Flow Model Calibration with Group-Sparsity Formulations
}

\author{
Azarang Golmohammadi ${ }^{1}$ and Behnam Jafarpour ${ }^{1,2}$
}

${ }^{1}$ Department of Electrical Engineering, University of Southern California

${ }^{2}$ Department of Chemical Engineering and Material Science, University of Southern California

Keywords:

Group-Sparsity, Model Calibration, Prior Geologic Scenario, Uncertainty, Inverse Modeling, Compressed Sensing

Corresponding author:

\author{
Behnam Jafarpour \\ Associate Professor \\ Viterbi School of Engineering \\ University of Southern California \\ HED 313, 925 Bloom Walk \\ Los Angeles, California, USA, 90089-1211 \\ (behnam.jafarpour@usc.edu)
}

February 14, 2016 


\begin{abstract}
Adopting representative geologic connectivity scenarios is critical for reliable modeling and prediction of subsurface flow and transport processes in subsurface environments. Geologic scenarios are often developed by integrating several sources of information, including knowledge of the depositional environment, qualitative and quantitative data such as outcrop and well $\operatorname{logs}$, and process-based geologic modeling. In general, flow and transport response data are usually not included in constructing geologic scenarios for a basin. Instead, these data are typically matched using a given prior geologic scenario as constraint. Since data limitations, modeling assumptions and subjective interpretations can lead to significant uncertainty in adopted geologic scenarios, flow and transport data may also be useful for constraining the uncertainty in proposed geologic scenarios. Constraining geologic scenarios with flow-related data opens an interesting and challenging research area, which goes beyond the traditional model calibration formulations where the geologic scenario is assumed given. In this paper, a novel concept, known as groupsparsity regularization, is proposed as an effective formulation to constrain the uncertainty in prior geologic scenario during subsurface flow model calibration. Given a collection of model realizations from several plausible geologic scenarios, the proposed method first applies the truncated singular value decomposition (TSVD) to compactly represent the models from each geologic scenario. The TSVD basis for representing each scenario forms a distinct group. The proposed approach searches over these groups (i.e., geologic scenarios) to eliminate inconsistent groups that are not supported by the observed flow/pressure data. The group-sparsity regularization consists of a $l_{2,1}$ mixed norm, where the $l_{2}$-norm quantifies the contribution of each group and operates on the coefficients within the groups while the $l_{1}$-norm is implemented
\end{abstract}


across the groups to select the significant groups. Numerical results demonstrate the effectiveness of the proposed method and its important properties.

\section{Introduction}

Prediction of flow and transport behavior in subsurface formations is critical for managing and controlling the development of the underlying water, energy and environmental resources. The spatiotemporal evolution of fluids within these formations is primarily governed by their underlying flow-related rock properties and the prevailing initial/boundary conditions [3]. A main difficulty in predictive modeling to describe the spatiotemporal behavior of these systems is the significant uncertainty in the distribution of flow properties such as permeability and porosity. In complex geologic settings, these properties can exhibit multi-scale heterogeneity, which further complicates their description. A major contributing factor to uncertainty in describing these properties stems from limited access to these formations for sampling. A common approach to constrain the uncertainty in describing the flow properties of subsurface formations is their calibration against dynamic flow data, typically accomplished through inverse modeling. A rich literature on subsurface flow model calibration techniques and their properties can be found in groundwater modeling and petroleum engineering (see, e.g., $[12,17,59,64,65,69$, $82,83])$.

Application of inverse modeling to characterization of subsurface environments has largely been challenged by the discrepancy between the desire to develop high resolution models and the limitation imposed by low spatial resolution and coverage of available data to adequately constrain such detailed models. This discrepancy often leads to ill-posed inverse problems in which an overwhelmingly large number of subsurface model parameters has to be estimated 
from scattered response data. Consequently, many geologically distinct solutions may be found that reproduce the observed data, but provide different predictions $[13,14,26,33,36,43,65]$. In general, additional information is needed to constrain the solutions to a geologically plausible set. Underdetermined inverse problems are solved by either reducing the number of model parameters, typically achieved through parameterization $[8,12,32,36,37,43,65,79]$, or by increasing the amount of information, often accomplished through regularization $[3,13,14,29$, $55,62,65,70,71,73,77,78]$. In both cases prior knowledge is used either implicitly, i.e., via information about solution structure (e.g., roughness) [78], or explicitly, using existing model(s) to constrain the solution $[13,14,51,52,59,65]$.

Parameterization methods attempt to reduce the number of parameters either in space domain, e.g., variants of zonation $[8,15,23,36,43]$ and pilot point methods $[18,30,54,67]$, or in a transform domain, most notably using compressive linear transform, e.g., Principle Component Analysis (PCA) [44,80], a.k.a., Karhunen-Leove Transform (KLT) [45,56], Discrete Cosine Transform (DCT) $[1,37,68]$, and Discrete Wavelet Transform (DWT) $[40,55,57,58]$. In transform domain parameterization methods, the solution is projected onto a predetermined or learned basis with desirable approximation properties that allow for a compact representation by identifying a subspace spanned by only a small subset of the basis elements. In traditional parameterization, such as the PCA, this subset is determined before inversion by selecting the leading eigenvectors of the parameter sample covariance matrix. In more recent formulations that originate from the compressed sensing paradigm [11,20], the approximation subspace is dynamically identified from the observed data during the inversion iterations, using an adaptive subspace selection mechanism [38,39,48,49,55]. A major difficulty in parameterization of subsurface flow 
properties is preserving complex geologic patterns, e.g., meandering channels, in parametric descriptions.

Regularization methods typically augment the data match requirement by penalizing solution deviation from a prior model or its stipulated attributes [29,73]. A classical example of regularization methods is the Tikhonov regularization [78] that promotes solution smoothness. Solution smoothness, however, is not a universal attribute and can result in oversimplification and unintended elimination of model complexity and heterogeneity $[60,61]$. Other regularization forms have also been proposed and applied in the past. A challenging aspect of regularization is going beyond mathematically convenient regularization forms and formulating more representative and geologically inspired regularization techniques that accurately prescribe and promote complex connectivity patterns. Recently, sparsity-based regularization techniques, inspired by the compressed sensing paradigm [11,20], that induce solution sparsity by minimizing its $l_{0}$ or $l_{1}$-norm have been introduced. In some cases, regularization and parameterization can be combined to effectively exploit certain characteristics of the solution, e.g., transform-domain sparsity [27,38,39,47-49,55].

In subsurface flow modeling, prior knowledge is available through quantitative and qualitative data, expert knowledge and interpretations, and process-based modeling of depositional environments [53]. Integration of these sources of information usually leads to a geologic continuity scenario, which is typically used as prior knowledge $[10,19,75]$. Traditionally, the adopted geologic scenario is used to constrain the solution of model calibration problems, for examples, by providing a variogram model [16] to describe the continuity in heterogeneous subsurface properties or a training image (TI) $[9,41,75]$ that prescribes the expected connectivity patterns in more complex geologic formations. Such prior models 
constrain the form of continuity in subsurface properties and allow for variability in the exact spatial locations of the existing patterns. A major flaw in adopting this approach is neglecting the uncertainty in the adopted geologic scenario, which can be quite significant. In general, provided with identical sources of information for a given geologic environment, different geologists may form different geologic scenarios and conceptual models of the formation that cannot be refuted. In fact, the scarcity of data from subsurface formations creates significant uncertainty in expert interpretations, which can lead to several plausible geologic scenarios that explain the available data. In general, without additional information, it may be impossible to discriminate against such proposed scenarios. Therefore, it is important to acknowledge and incorporate the uncertainty in geologic continuity model, i.e. model structure and geologic scenario, in solving model calibration inverse problems $[42,49,50,70,71]$.

An important implication of adopting a geologic scenario prior to model calibration is that flow and monitoring data are not used to constrain it, resulting in an opportunity loss to potentially correct geologic scenarios that are not supported by data. Therefore, an interesting problem is to incorporate the flow data into prior geologic scenario selection $[27,49]$. When a set of geologic scenarios are proposed as prior knowledge, one could implement a model calibration using each scenario and generate a set of feasible solutions depending on the prior geologic scenario. However, this approach requires multiple model calibration runs, which is not feasible when several possible scenarios are proposed. Additionally, this method does not provide any insight into possible modification of the proposed models, for example when the true geologic scenario is not consistent with any of the proposed models or when multiple prior models should be combined to capture the existing features. An alternative approach that is proposed in this paper is to develop a model calibration formulation that can simultaneously incorporate several 
prior geologic scenarios and, in addition to identifying a calibrated model, can automatically select geologic scenarios that are supported by flow response data. The flow and transport related data types include steady state and transient pressure data, phase flowrates and saturations, tracer data, etc. These flow/transport-relevant data are usually employed to calibrate a given geologic scenario; they are typically not used for constraining uncertain geologic scenario.

To formulate a model calibration framework to identify consistent prior geologic scenarios, we take advantage of the group-sparsity regularization using mixed $l_{1} / l_{2}$ norm $[4,27$, $63,66]$. The mixed $l_{1} / l_{2}$-norm penalty provides an effective regularization form to recover blocksparse signals $[22,35,74]$. Block-sparse signals are a subset of sparse signals whose components are classified into predefined groups of variables with the following properties [22]: (1) all the elements within a group are expected to be either collectively active (non-zero) or inactive (zero); (2) only a minimum number of groups can be active (hence the group-sparse property). In promoting group-sparsity with $l_{1} / l_{2}$-norm, the $l_{2}$-norm is applied to elements within each group (to represent the contribution of that group) while the $l_{1}$-norm operates across the groups to promote sparsity (minimum number of active groups) [34,66].

To screen geologic scenarios with group-sparsity, we apply the $l_{2}$-norm to first quantify the contribution of each group (geologic scenario) and then apply the $l_{1}$-norm across the groups to promote sparsity. The grouping is achieved through a truncated SVD (TSVD) parameterization of model realizations for each geologic scenario prior to model calibration. To this end, each proposed geologic scenario is first used to generate an ensemble of model realizations (e.g., using geostatistical simulation techniques). The simulated models within each group are used to construct a TSVD approximation basis for each scenario. The $l_{2}$-norm of the TSVD coefficients within each group quantifies the contribution of that group. Once the 
contribution of each group is computed, group-sparsity is promoted by minimizing the $l_{1}$-norm of the quantified contributions of the groups, which is known to have sparsity-inducing property. This ensures that only groups (geologic scenarios) that have significant contributions to the solution will be retained and irrelevant groups are eliminated. This formulation is implemented by minimizing a regularized inversion objective function that consists of a flow response data mismatch term and a mixed $l_{1} / l_{2}$-norm regularization term to remove inconsistent geologic scenarios.

The main contribution of this paper is the development of a simultaneous model calibration and prior geologic scenario identification method to address an important challenge in subsurface flow modeling. The developed method is capable of incorporating several uncertain prior geologic scenarios, and selecting the ones that have significant contribution to reproducing the observed flow/pressure data. The implementation details and important properties and behavior of the developed model calibration techniques are presented and discussed in the remainder of the paper. In Section 2, we briefly review the TSVD representation of each geologic scenario, followed by a detailed discussion of the mixed $l_{1} / l_{2}$-norm regularization. We then present the group-sparsity regularization in presence of multiple prior geologic scenarios, including training images and variogram models. Section 3 provides a series of numerical experiments to evaluate the performance of the presented method. Concluding remarks about the application and implication of the developed method are summarized before closing the paper. 


\section{Methodology}

In this section, we present the group-sparsity formulation and its application to simultaneous geologic scenario selection and model calibration. A summary of our main notations is presented first. We denote the $q$-norm of a length- $n$ vector, $\mathbf{u}=\left[u_{1}, u_{2}, \ldots, u_{n}\right]^{T}$, as $\|\mathbf{u}\|_{q}(q>0)$, where

$$
\|\mathbf{u}\|_{q}=\left(\sum_{i=1}^{n}\left|u_{i}\right|^{q}\right)^{\frac{1}{q}}
$$

and the operator $|$.$| returns the absolute value of its argument. For a matrix \mathbf{A}$, the Frobenius norm $\|\mathbf{A}\|_{F}^{2}$ is defined as the sum of square of its entries, for which the relation $\|\mathbf{A}\|_{F}^{2}=\operatorname{tr}\left\{\mathbf{A} \mathbf{A}^{T}\right\}$ holds. In formulating our inverse problem, we consider a $n$-dimensional model parameter vector $\mathbf{u}_{n \times 1}$, and a $m$-dimensional observation vector $\mathbf{d}_{m \times 1}$ (flow/pressure data), with a general forward model $\mathbf{g}($.$) that relates model parameters to data, that is \mathbf{g}(\mathbf{u})=\mathbf{d}$ (in the absence of any data noise). The Jacobian matrix $\mathbf{G}_{m \times n}$ summarizes the partial derivatives of model outputs (at measurement locations) with respect to unknown model input parameters (flow properties everywhere in the domain), that is, the entry $(i, j)$ of this matrix can be written as $\mathrm{G}_{i, j}=\frac{\partial g_{i}(\mathbf{u})}{\partial u_{j}}$.

A general linear approximation of the parameters of interest $\mathbf{u}$ in an incomplete (truncated) basis of dimension $\ll n$, i.e., $\boldsymbol{\Phi}=\left\{\Phi_{1}, \Phi_{2}, \ldots, \Phi_{s}\right\}$, can be expressed as:

$$
\mathbf{u} \approx \sum_{i=1}^{S} \Phi_{i} v_{i}=\mathbf{\Phi} \mathbf{v}
$$

where $\mathbf{v}=\left[v_{1}, v_{2}, \ldots, v_{s}\right]$ is the vector of expansion (approximation) coefficients that represent the importance (weight) of each basis function in approximating $\mathbf{u}$. Smaller coefficients (in magnitude) indicate less significant contribution of the corresponding basis element in the approximation. The choice of $\boldsymbol{\Phi}$ is problem-specific and depends on the expected properties and features in the solution, as well as the inversion formulation. In addition, the $s$ expansion functions define the approximation subspace that must be selected from a set of $n$ (complete) 
basis components. In traditional parameterization methods, such as PCA or DCT, this subspace selection is done a-priori based on available prior knowledge about the parameters. For example, in the PCA method, for a rank- $s$ approximation the subspace is defined by the first $s$ leading eigenvectors of the sample parameter covariance matrix, i.e., $\mathbf{C}_{\mathbf{u}}$. Therefore, the inversion data $\mathbf{d}$ do not play a role is identifying the approximation subspace; instead, its primary function is to estimate the expansion coefficients for a predetermined approximation subspace. Recently, however, advances in sparse signal processing and compressed sensing have introduced efficient methods for adaptive optimal subspace selection based on the inversion data d. Sparse reconstruction methods look for minimum support expansions (i.e., approximation subspace dimension) by minimizing the $l_{0}$-norm (or alternatively $l_{1}$-norm) of the complete expansion coefficients $\mathbf{v}$ to adaptively select the best subspace using the observed data.

Mathematically, the solution of the inverse problem in the expansion domain is found by minimizing a regularized objective function of the form

$$
\min _{\mathbf{v}} J(\mathbf{v})=\left\|\mathbf{C}_{\mathbf{d}}^{-1}(\mathbf{d}-\mathbf{g}(\boldsymbol{\Phi} \mathbf{v}))\right\|_{2}^{2}+\alpha S P(\mathbf{v})
$$

where $S P(\mathbf{v})$ is a sparsity-inducing regularization term, which is often expressed as $\|\mathbf{v}\|_{0}$ or $\|\mathbf{v}\|_{1}$; the regularization parameter $\alpha$ adjusts the tradeoff between data misfit and regularization terms, and $\mathbf{C}_{\mathbf{d}}^{-1}$ is typically a diagonal matrix that contains the inverse of data noise variance in its diagonal entries [3]. As in other regularized inversion methods, determining the regularization parameter $\alpha$ presents a hurdle. In linear inverse theory, well-established methods such as the L-curve [3,31], cross validation [3,30], or more recently LARS [21] (for sparse reconstruction) methods are available to guide the choice of $\alpha$. Unfortunately, these methods are limited to linear problems and do not extend to more general nonlinear problems that are encountered in practice. Additionally, for nonlinear problems with computationally 
demanding forward runs, these methods require hundreds of simulation, making them impractical. In such cases, partial cross-validation and trial and error are practical ways for determining a reasonable value for $\alpha$.

The singular value decomposition (SVD) is a widely used method for solution of inverse problems [28]. The SVD method has also been used to learn an approximation basis from prior model by applying it to the matrix that has the prior realizations of the parameter vectors in its columns. After truncation of non-leading leading left singular vectors, the remaining left singular vector for an approximation basis known as truncated SVD (or TSVD). Stacking $r$ prior realizations of the parameter vector, $\mathbf{u}_{i=1: r}$, into a matrix $\mathbf{U}$, i.e., $\mathbf{U}=\left[\mathbf{u}_{1}, \mathbf{u}_{2}, \ldots, \mathbf{u}_{r}\right]$, and applying the SVD to it yields a set of left singular vectors, of which the leading $s$ elements can provide an orthogonal approximation basis $\boldsymbol{\Phi}=\left[\boldsymbol{\phi}_{1}, \boldsymbol{\phi}_{2}, \ldots, \boldsymbol{\phi}_{s}\right]$. The resulting basis minimizes the following objective function:

$$
\min _{\boldsymbol{\Phi}, \mathbf{\Lambda}, \mathbf{V}} J(\boldsymbol{\Phi}, \boldsymbol{\Lambda}, \mathbf{V})=\|\mathbf{U}-\boldsymbol{\Phi} \mathbf{\Lambda} \mathbf{V}\|_{F}^{2}
$$

where, $\boldsymbol{\Lambda}=\operatorname{diag}\left\{\lambda_{1}, \lambda_{2}, \ldots, \lambda_{s}\right\}$ is a diagonal matrix holding the singular values in its diagonal elements, and $\mathbf{V}$ contains the coefficient vectors (normalized by the singular values) corresponding to the prior training data $\mathbf{U}$ in the learned basis $\boldsymbol{\Phi}$.

While the SVD basis has excellent approximation properties when reliable prior models are used, one of its major weaknesses is its sensitivity to the quality of the prior model $[37,48]$. For instance, structurally incorrect prior training data can significantly reduce the approximation efficiency of the TSVD basis. In particular, if two sets of structurally distinct prior models are used for training the TSVD basis, the resulting basis elements lose the distinct features of each set and essentially produce a basis with features that do not represent the distinct prior models, and instead aggregates the distinct features. Hence, the use of TSVD for approximation of prior 
models with uncertain connectivity patterns, such as multiple variograms or training images, is not advisable. The central topic of this paper is how to deal with multiple prior models that represent different geologic scenarios in solving an inverse problem. To address this problem, we apply the TSVD parametrization to each set of prior models (to preserve their distinct continuity) and, during inversion, invoke the mixed $l_{1} / l_{2}$-norm regularization to discriminate against distinct prior based on available dynamic/static data, and without losing the main features of each geologic scenario.

\subsection{Mixed $l_{1} / l_{2}$-Norm for Group Sparsity Regularization}

Consider a length- $n$ group sparse signal $\mathbf{v}$ that is composed of $p$ distinct groups of coefficients $\mathbf{v}_{i=1: p}$, i.e., $\mathbf{v}=\left[\mathbf{v}_{1}^{T}, \mathbf{v}_{2}^{T}, \ldots, \mathbf{v}_{p}^{T}\right]^{T}$, where only $z \ll p$ groups can be active (have nonzero coefficients). In general, the groups can have overlapping elements, with union $\mathbf{v}$. While this signal is clearly a sparse signal, it has an additional property, its sparsity has a group structure, that is, groups of coefficients are collectively active or inactive. The group-sparse structure (which, in our application, will be ensured by construction) can indeed provide additional constraining power to help with signal reconstruction from incomplete observations. If the $l_{2}$ norm is used to define the energy contribution of group $(i)$ coefficients, i.e. $w_{i}=\left\|\mathbf{v}_{i}\right\|_{2}=$ $\left(\sum_{k=1}^{s_{i}}\left|v_{k}\right|^{2}\right)^{\frac{1}{2}}$, then $\mathbf{w}=\left[w_{1}, w_{2}, \ldots, w_{p}\right]^{T}$ is a sparse vector. Hence, estimating the group-sparse signal $\mathbf{v}$ can be accomplished by regular sparsity-promoting algorithms for $\mathbf{w}$, which can be achieved through celebrated $l_{1}$-norm minimization [34]. Hence, the resulting regularization function takes the form

$$
\|\mathbf{w}\|_{1}=\sum_{i=1}^{p}\left|w_{i}\right|=\left|w_{1}\right|+\left|w_{2}\right|+\cdots+\left|w_{p}\right|
$$

which after substituting for $w_{i}=\left\|\mathbf{v}_{i}\right\|_{2}$, leads to the mixed $l_{1} / l_{2}$-norm regularization function 


$$
\|\mathbf{w}\|_{1}=\|\mathbf{v}\|_{1,2}=\left\|\mathbf{v}_{1}\right\|_{2}+\left\|\mathbf{v}_{2}\right\|_{2}+\cdots+\left\|\mathbf{v}_{p}\right\|_{2}=\sum_{i=1}^{p}\left\|\mathbf{v}_{i}\right\|_{2}
$$

Minimizing the above mixed $l_{1} / l_{2}$-norm regularization along with a data mismatch term, i.e.,

$$
\min _{\mathbf{v}} J(\mathbf{v})=\left\|\mathbf{C}_{n}^{-1}(\mathbf{d}-\mathbf{g}(\mathbf{v}))\right\|_{2}^{2}+\alpha \sum_{i=1}^{p}\left\|\mathbf{v}_{i}\right\|_{2}
$$

promotes group-sparse solutions, which is a stronger condition than $l_{1}$-norm sparsity regularization. The above formulation assumes that the groups are equally important. If prior information is available to suggest different weights for the groups, and also for each basis elements within, the formulation can be generalized into

$$
\min _{\mathbf{v}} \quad J(\mathbf{v})=\left\|\mathbf{C}_{n}^{-1}(\mathbf{d}-\mathbf{g}(\mathbf{\Phi} \mathbf{v}))\right\|_{2}^{2}+\alpha \sum_{i=1}^{p} \mu_{i}\left\|\mathbf{K}_{i} \mathbf{v}_{i}\right\|_{2}
$$

where $\mu_{i}$ is the relative weight given to the $i^{\text {th }}$ group and $\mathbf{K}_{i}^{T} \mathbf{K}_{i}$ is a diagonal weight matrix for elements within the $i^{\text {th }}$ group. In the absence of any prior information, the weights $\mu_{i}$ and $\mathbf{K}_{i}^{T} \mathbf{K}_{i}$ can be set to 1 and identity matrix, respectively. Appendix A presents an iteratively reweighted algorithm for solving the optimization problem in Eq. (8).

A simple example can illustrate the main distinction between the mixed $l_{1} / l_{2}$-norm and the regular $l_{1}$-norm minimizations. Consider the unknown vector of parameters $\mathbf{v}=\left[\begin{array}{lll}v_{1} & v_{2} & v_{3}\end{array}\right]$ that are grouped into $\mathbf{v}_{1}=\left[v_{1}, v_{2}\right]$ and $\mathbf{v}_{2}=\left[v_{3}\right]$. Based on this grouping, the mixed $l_{1} / l_{2}$ and regular $l_{1}$ norms are defined as $\left(v_{1}^{2}+v_{2}^{2}\right)^{\frac{1}{2}}+\left|v_{3}\right|$ and $\left|v_{1}\right|+\left|v_{2}\right|+\left|v_{3}\right|$, respectively. Figure 1 depicts the geometric interpretations of $\left(v_{1}^{2}+v_{2}^{2}\right)^{\frac{1}{2}}+\left|v_{3}\right|=c_{1}$ and $\left|v_{1}\right|+\left|v_{2}\right|+\left|v_{3}\right|=c_{1}$. For linear measurement constraints, that is, $\mathbf{d}=\mathbf{G v}$, the solution should lie at the intersection of a hyperplane with the balls shown in Figure 1 . With the $l_{1}$-norm penalty a sparse solution is more likely to occur on one of the axes (Figure $1 \mathrm{~b}$ ). On the other hand, for the $l_{1} / l_{2}$ function, the solution does not have to occur on one of the axes since $v_{1}$ and $v_{2}$ can simultaneously assume 
non-zero values at the minimum. Hence, group-sparsity allows for parameters within each group to take non-zero values without affecting the regularization term.

Although the group-sparsity regularization can be applied to reconstruct group sparse signals [63], an important assumption is the knowledge of grouping and group sparse structure. To compare the performance of group-sparsity with regular $l_{1}$-norm sparsity regularizations, Figure 2 shows the reconstruction results for a group-sparse signal. In this example, the groupsparse signal $\mathbf{v}$ has 150 entries that are clustered into 10 groups of 15 consecutive elements (Figure 2a). Only Groups 3 and 8 are active in the reference model. A linear measurement of the form $\mathbf{d}=\mathbf{G} \mathbf{v}$ is assumed, where $\mathbf{G}$ has entries drawn from an independent and identically distributed Gaussian probability density function [11]. We use the resulting random measurements (not shown) to evaluate the reconstruction results in a linear least-square formulation containing a data mismatch term $\|\mathbf{d}=\mathbf{G v}\|$ with regular sparsity and group-sparsity regularization to highlight the different behavior of the two. Figures 2b1-2b5 depict the reconstruction results using $l_{1} / l_{2}$ regularization (left) and $l_{1}$ regularization (right) with 10,20 , 40, 60 and 80 measurements, respectively. When fewer observations are available, such as in subsurface flow model calibration problems, group-sparsity provides a superior constraining power to regular $l_{1}$-norm sparsity regularization. The two methods give similar results when a large number of observations is used.

To explore the effect of incorrect grouping, Figure 3 shows the results of two sets of experiments. In the first set, Figures 3a1-3a5 (left column), 30 groups with 5 elements in each group are assumed (each two consecutive group is equivalent to one group in the original example). The results show departure from the reference model, especially when fewer observations are used. In the second case, Figures 3b1-3b5, each group contains 10 elements 
with completely misplaced grouping structure. In this case, several irrelevant elements are also selected, especially when fewer observations are used. These two experiments highlight the importance of consistent grouping in successful implementation of group-sparsity regularization.

Another important factor in the group-sparsity formulation is the regularization parameter $\alpha$. In addition to controlling the final solution, the value of $\alpha$ can have an impact on the selected groups, which can have significant implication in our geologic scenario selection application. Figure 4 shows the sensitivity of selected groups to the regularization parameter $\alpha$ in the range $\left[10^{-15}, 800\right]$, when 60 observations are used. Intuitively, for very small (large) values of $\alpha$, the regularization (data mismatch) term is neglected, resulting in overfitting (underfitting) of data. However, the behavior of the solution for midrange $\alpha$ values is not easy to predict. Interestingly, the selected groups show little sensitivity to the specified $\alpha$ value within a large range. It appears that the variability in $\alpha$ only affects the magnitude of the selected coefficients without changing the selected groups. Figure $4 \mathrm{~b}$ displays the data match for the solutions obtained with different values of $\alpha$, which shows better data matches as $\alpha$ decreases. These results suggest that, with a wide range, the group selection property of $l_{1} / l_{2}$ regularization is robust against the choice of $\alpha$.

Next, we take advantage of the group selection property of the $l_{1} / l_{2}$ regularization to formulate a simultaneous geologic scenario identification and subsurface flow model calibration approach. The primary objective is to simultaneously perform model calibration and identify consistent geologic scenario(s) from several proposed plausible scenarios. A natural way to proceed is to place each geologic scenario into a group, as discussed below. 


\subsection{Geologic Scenario Identification with Group-Sparse Formulations}

A significant source of uncertainty that can bias model calibration and future predictions can originate from the adopted geologic scenario, which is often derived from available data with considerable interpretation and subjectivity. It is not uncommon for different geologists to provide alternative conceptual models of connectivity when the available data is very limited. However, despite its significant impact, the uncertainty in the geologic scenario, which is responsible for the global flow behavior, is usually neglected in formulation of model calibration $[42,49,50]$. In this section, a group-sparsity formulation is used to develop to constrain geologic scenario with flow data. We use several realizations from a distinct geologic scenario to form a group that represents the respective geologic scenario. While geologic scenarios are conceptual and qualitative representations of connectivity, process-based geologic modeling can be applied to transform such conceptual models into quantitative representations. Furthermore, geostatistical simulation techniques are available to integrate the resulting geologic scenarios (e.g., training images) with available data to derive many conditional realizations that represent the likely distribution of rock formations.

Given $p$ plausible prior geologic scenarios that are proposed for model calibration, each scenario is first used to generate an ensemble if model realization (typically using geostatistical methods). Collecting the realizations for geologic scenario $(i)$ in columns of a matrix $\mathbf{U}_{i}$ results in $\mathbf{U}_{i}$ such matrices. i.e., $\mathbf{U}_{1}=\left[\mathbf{u}_{11} \mathbf{u}_{12} \ldots \mathbf{u}_{1 N}\right], \mathbf{U}_{2}=\left[\mathbf{u}_{21} \mathbf{u}_{22} \ldots \mathbf{u}_{2 N}\right], \ldots, \mathbf{U}_{p}=\left[\mathbf{u}_{p 1} \mathbf{u}_{p 2} \ldots \mathbf{u}_{p N}\right]$. Prior to model calibration, a compact representation of model realizations in each group, $\mathbf{U}_{i}$, can be obtained by applying the TSVD parameterization to each group and forming the basis $\boldsymbol{\Phi}_{i}$ (of dimension $n \times s_{i}$ ). Note that, the truncation level $s_{i}$ can be different for different groups, to account for the variable complexity of the geologic scenarios. A typical approach is to choose the 
number of singular vectors to maintain a certain fraction of the total variance (energy) in the original model, which determines the desired level of approximation. In addition, the number of realizations in each prior set should be sufficient to capture the variability and complexity of the connectivity model that they represent. A simple procedure to ensure that the included realizations are sufficient is cross-validation where the TSVD parameterization is used to approximate members from each group that are intentionally left out of the training set that used to construct the singular vectors. Combining the TSVD basis for each group leads to a hybrid basis $\boldsymbol{\Phi}=\left[\boldsymbol{\Phi}_{1}, \boldsymbol{\Phi}_{2}, \ldots, \boldsymbol{\Phi}_{p}\right]=\left[\boldsymbol{\Phi}_{n \times s_{1}}, \boldsymbol{\Phi}_{n \times s_{2}}, \ldots, \boldsymbol{\Phi}_{n \times s_{p}}\right]$. Hence, a typical model from these groups can be approximated as

$$
\mathbf{u}=\boldsymbol{\Phi}_{\mathrm{g}} \mathbf{v}_{\mathrm{g}}=\left[\begin{array}{llll}
\boldsymbol{\Phi}_{1} & \boldsymbol{\Phi}_{2} & \ldots & \boldsymbol{\Phi}_{p}
\end{array}\right]\left[\begin{array}{lll}
\mathbf{v}_{1}^{T} & \mathbf{v}_{2}^{T} & \ldots \\
\mathbf{v}_{p}^{T}
\end{array}\right]^{T}
$$

where $\boldsymbol{\Phi}_{\mathrm{g}}$ denotes the combined dictionary, $\left[\mathbf{v}_{1}^{T} \mathbf{v}_{2}^{T} \ldots \mathbf{v}_{p}^{T}\right]^{T}$ is a $\left(\sum_{i=1}^{p} s_{i}\right) \times 1$ vector that contains the expansion coefficients in representing $\mathbf{u}$. Hence, the vector of $\mathbf{v}_{i, 1 \leq i \leq p}=\left[v_{i 1}, v_{i 2}, \ldots, v_{i s_{i}}\right]^{T}$ contains the representation coefficients for basis elements within each group, $\boldsymbol{\Phi}_{i, 1 \leq i \leq p}$. With this definition, if the groups are distinct and the solution belongs to one of the groups, the coefficients corresponding to the basis elements within that group will have significantly higher contribution to the expansion. It is possible for more than one single group to have significant contribution to the solution; however, if the groups are distinct, it is less probable for many groups to contribute to the solution, implying the desired group-sparsity property.

In this paper, we consider the $l_{2}$-norm of the expansion coefficients for each group as a measure of relative contribution (importance) of the corresponding initial geologic scenarios. Hence, groups with larger $l_{2}$-norm (of coefficient) have larger contributions to the final solution and correspond to a higher probability for the corresponding geologic scenario. However, alternative model selection criteria are available [24,81], and can be applied. For example, 
information based techniques such as Akaike Information Criterion (AIC) [2] and its small sample version $\mathrm{AIC}_{\mathrm{c}}$ [35], Bayesian based methods such as Bayesian Information Criterion (BIC) [76] and Kashyap's Information Criterion (KIC) [46], have also been used for mdel selection. In these cases, $\mathbf{u}_{i}=\boldsymbol{\Phi}_{i} \mathbf{v}_{i}$ is the contribution of the $i^{\text {th }}$ geologic scenario in representing the final solution in $\mathbf{u}=\left[\begin{array}{lllll}\boldsymbol{\Phi}_{1} & \boldsymbol{\Phi}_{2} & \ldots & \boldsymbol{\Phi}_{p}\end{array}\right]\left[\begin{array}{llll}\mathbf{v}_{1}^{T} & \mathbf{v}_{2}^{T} & \ldots & \mathbf{v}_{p}^{T}\end{array}\right]^{T}$. Hence, comparing $\mathbf{u}_{i}=$ $\boldsymbol{\Phi}_{i} \mathbf{v}_{i}$ with $\mathbf{u}$ based on the measurement criteria defined in these methods can provide a probabilistic assessment of the contribution of each geologic scenario. In these methods, inactive (active) groups will result in a larger (smaller) error, and hence, the measurement criteria of these methods assign a smaller (larger) probability to the corresponding geologic scenarios. It is, however, important to note that our formulation does not treat the geologic scenarios independently, and considers the solution as a sparse linear combination of the assumed geologic scenarios.

With the above grouping of geologic scenarios, a group-sparse formulation, e.g. Eq. (8), can be invoked to identify consistent geologic scenarios from a set of uncertain prior models. In the TSVD representation, the singular vectors within each group are ordered based on the magnitude of the singular values, which can provide a reasonable choice for the weights matrix $\mathbf{K}_{i}^{T} \mathbf{K}_{i}$, which will be given by the diagonal matrix $\left(\mathbf{\Lambda} \mathbf{\Lambda}^{T}\right)^{-1}$. With this choice, during inversion the leading elements of the TSVD basis (with larger singular values) are penalized less harshly. To illustrate the effectiveness of group-sparsity regularization in presence of multiple distinct prior geologic scenarios, we consider the three TIs in Figure 5a that represent different types of connectivity structures. Assuming that the direction of channel continuity is unknown, Figure 5b shows a total of 10 different scenarios (groups). Note that for the meandering channels only two directions are selected, i.e., $\theta=0^{\circ}$ and $\theta=90^{\circ}$, while for the non-meandering channels, four 
distinct channel directions are considered, i.e., $\theta=0^{\circ}, 45^{\circ}, 90^{\circ}, 135^{\circ}$. Figure $5 \mathrm{~b}$ depicts four samples realizations (out of $N=500$ ) for each set. These realizations are generated from the corresponding TIs using the Single Normal Equations Simulation (SNESIM) algorithm [75], with the specified global rotations. Figure 5c shows the first 25 leading TSVD basis elements for each geologic scenario. The plot to the right in Figure 5c displays the leading TSVD basis elements, denoted as $\boldsymbol{\Phi}_{\mathrm{m}}$, that would be obtained if all the prior model realizations with different geologic scenario were mixed before implementing SVD. While the individual bases $\boldsymbol{\Phi}_{i, 1 \leq i \leq 10}$ preserve the main geologic features for each scenario, the mixed basis $\boldsymbol{\Phi}_{\mathrm{m}}$ tends to aggregate geologic features from distinct scenarios, resulting in a loss of channel connectivity.

An important aspect of the TSVD representation is the number of retained basis elements. This choice typically depends on the decay rate of the singular values that contain the variance (energy) of the underlying spatial images. To retain the same level of variance in a truncated approximation, complex geologic features such as meandering channels tend to require more expansion terms that straight channels. Figure 6a plots, for samples from two geologic scenarios, the cumulative energy against the number of retained basis elements. Since the meandering channel feature is more complex than the intersecting channels, for the same energy level a larger number of basis elements are needed to represent the meandering channel. Figure $6 \mathrm{~b}$ shows the approximation quality for the two cases using different energy levels. In this paper, we chose to retain $80 \%$ of the energy in truncating the TSVD bases for each group.

The central part of the group-sparsity formulation is the mixed $l_{1} / l_{2}$ norm minimization. The use of this mixed norm implies that, amongst all the groups proposed, the correct group provides the lowest $l_{2}$-norm for the same approximation quality. This is explored in Figure $\mathbf{7}$, where for a sample model from the first (top) and fourth (bottom) groups, the best approximation 
with each geologic scenario is shown on the left. The corresponding root-mean-squared-error (RMSE) and $l_{2}$-norm of the expansion coefficients are also displayed as bar plots. In each case, the best data match is achieved by the correct group. To further explore the robustness of group sparsity algorithm, Figures 8a though 8c (first column) depict three reference permeability fields that are approximated (compressed) using 10 prior geologic scenarios in Figure 5. Hence, in this example, the observed data are the parameter values at all grid blocks $(\mathbf{d}=\mathbf{u})$ and $\mathbf{g}(\boldsymbol{\Phi} \mathbf{v})=\boldsymbol{\Phi} \mathbf{v}$ in Eq. (8). Also, in this case, no noise is added to the observed data. Figure 8a shows the reference and best achievable permeability map for the case where the connectivity structure is consistent with those in Group 4 (intersecting channels in $\theta=45^{\circ}$ direction). In this case, groupsparsity is able to pick the basis elements in the correct group. In Figure 8b, the reference model has both intersecting channel features in directions $\theta=90^{\circ}$ and $\theta=135^{\circ}$. From the reconstruction results, the active bases are from Group 4 and Group 6, which is consistent with the features in the reference model. Finally, Figures $8 \mathrm{c}$ shows a multi-Gaussian reference permeability model, which clearly does not belong to any of the groups. The reconstructed coefficients show that several groups are selected to provide a rough approximation for this model, suggesting that a group-sparse formulation is not appropriate. This typically indicates that a similar solution cannot be obtained by using only a small subset of prior models. One approach to resolve this problem is to revise the initial geological scenarios, based on the obtained results as well as additional geologic input, and repeat the solution.

To investigate the behavior of the misfit and regularization terms in the objective function Figure 9 shows, with different initializations, the evolutions of these functions along with the expansion coefficients, the resulting reconstruction results, and the contribution $\left(l_{2}\right.$ norm $)$ of each group to the reconstruction. Three different initializations are shown for each example: very 
small (Case 1), very large (Case 2), and randomly assigned (Case 3) coefficients (i.e., regularization term). While the final solution and the selected groups are the same, the behavior of the regularization and misfit terms depends on the initialization. When the initial value of the regularization term is small (Case 1), this term is first increased by activating a few potential groups and later decreased by keeping only the consistent group. On the other hand, when the regularization term is initially large and has several active groups (Case 2), the algorithm tends to reduce the regularization term by eliminating groups with insignificant contributions. In Case 3, the solution is initialized with one inconsistent active group, resulting in a large regularization term. In this case, the regularization term is also decreased by reducing the contribution of the inconsistent group while slightly activating other groups. The regularization term will continuously decrease by eliminating irrelevant groups and identifying the consistent geologic scenario. A similar behavior is observed with several other examples, which is also in agreement with the discussion in the previous section. Overall, regardless of the initialization, the solution algorithm first activates a relatively large number of basis elements (groups) and then selects the groups that provide a better match to data and a minimum length. At later iterations, the group selection is stabilized and the coefficients are refined around the final selected group(s). A pseudo code of overall inversion procedure can be found in Appendix B.

\section{Numerical Experiments}

Two sets of numerical examples will be discussed in this section to examine the effectiveness of group-sparsity regularization for geologic scenario identification. The first experiment involves a travel-time tomographic inversion as a linear inverse problem while the second example consists of a groundwater well pumping test in which the transmissivity of an aquifer is estimated from 
pressure and transmissivity observations. For the pumping test, three separate examples are discussed, a channel type transmissivity field in a 2D aquifer, a 3D test case with a multiGaussian transmissivity field and another 3D example with channel type structures. The results are presented and compared for group-sparsity (mixed $l_{1} / l_{2}$-norm) and regular $l_{1}$-norm sparsity regularizations.

\subsection{Tomographic Inversion}

In straight ray tomography, the slowness (1/velocity) map of a subsurface environment is inferred from observations of acoustic wave travel time on straight paths (see [5-7] for forward equations). Figure 10a shows the configuration of this example with a set of transmitter (left) and receiver (right) arrays. A $1000 \times 1000 \mathrm{~m}^{2}$ domain is discretized into $100 \times 100$ cells of dimensions $10 \times 10 \mathrm{~m}^{2}$. Two sets of experiments are presented, one with multi-Gaussian reference model and prior geologic scenarios (variogram), and a second with complex channel connectivity patterns in the reference and prior geologic scenarios (i.e., TIs). In both cases, 8 transmitters and 8 receivers are used, resulting in 64 arrival time observations. Figures $10 \mathrm{~b}$ and 10c display the parameters of the prior variogram models and their corresponding model realizations.

\section{Case 1: Multi-Gaussian Slowness Map}

This example is used to demonstrate the application of the proposed method to distinguish variogram models that control the connectivity in flow properties. The reference slowness map for this case is shown in Figure 11a (top left). This map is generated using sequential Gaussian simulation with the variogram model $y_{2}(h)$ in Figure 10b. The anisotropy direction in the 
reference model is $\theta=45^{\circ}$, however the variogram model parameters and the direction of maximum continuity is unknown. Hence, prior model realizations are generated using three variogram models $y_{1}(h), y_{2}(h)$ and $y_{3}(h)$ (Figure 10b), each with four anisotropy directions, $\theta=0^{\circ}, 45^{\circ}, 90^{\circ}, 135^{\circ}$, resulting in a total of 12 variogram models (groups). Each variogram is used to generate 500 prior realizations, which are vectorized and used as columns of matrices $\mathbf{U}_{1}, \ldots, \mathbf{U}_{12}$ (see Figure 10c for sample realizations). These realizations are conditioned on hard data; however, other data types such as tomographic ray arrival times are not incorporated and will be used for variogram identification. The TSVD parameterization is then separately applied to each group to construct the 12 different bases that are combined into $\boldsymbol{\Phi}_{\mathrm{g}} \in$ $\mathrm{R}^{10000 \times 1076}$. In each group, the number of retained basis elements for the parameterization is selected to preserve $80 \%$ of the variance within that group. Also, to construct $\boldsymbol{\Phi}_{\mathrm{m}}$, the SVD decomposition is performed after mixing the realizations from all groups, and the leading 100 basis components are selected. The left column in Figure 11a displays the reference model (top) and the best achievable solutions with $\boldsymbol{\Phi}_{\mathrm{g}}$ (middle) and $\boldsymbol{\Phi}_{\mathrm{m}}$ (bottom).

The correct variogram model for this example belongs to Group $\mathbf{U}_{6}$. The reconstruction results with the $l_{1} / l_{2}$-norm minimization mainly picks $\boldsymbol{\Phi}_{6}$ as the relevant basis with some active elements from the $\boldsymbol{\Phi}_{7}$ basis. This suggests that some of the elements in $\boldsymbol{\Phi}_{7}$, have important contribution to reproducing the data. Note that the anisotropy ratio for $\boldsymbol{\Phi}_{6}$ and $\boldsymbol{\Phi}_{7}$ is the same; however, these groups have different directions of anisotropy. Also, the top left corner of the reference map contains similar features to those in $\boldsymbol{\Phi}_{7}$. When the $l_{1}$-norm regularization is used instead of $l_{1} / l_{2}$-norm (middle row), the reconstruction algorithm selects several basis elements from different (and inconsistent) variograms, lacking a major contribution from the consistent basis $\boldsymbol{\Phi}_{6}$. Although the estimated parameter in this case is similar to the case with the $l_{1} / l_{2}$-norm 
regularization, the model selection property is not observed in this case. The reconstruction results with $\boldsymbol{\Phi}_{\mathrm{m}}$ (obtained by aggregating all prior models) does not reveal the main continuity feature in the reference model, demonstrating the sensitivity of the TSVD parameterization to prior model uncertainty.

\section{Case 2: Channel-Type Slowness Map}

The reference slowness map in this case is shown in Figure 11b (top left). The low (blue regions) and high (red regions) slowness values are 0.5 and $1 \mathrm{~ms} / \mathrm{m}$, respectively. The prior TIs and their corresponding realizations are depicted in Figure 5. The TSVD parametrization is designed to preserve $80 \%$ of energy within each prior model. The group-sparsity basis consists of 466 elements, i.e., $\boldsymbol{\Phi}_{\mathrm{g}}=\left[\boldsymbol{\Phi}_{1} \boldsymbol{\Phi}_{2} \ldots \boldsymbol{\Phi}_{10}\right] \in \mathrm{R}^{10000 \times 466}$. For the basis generated from the mixed realizations, $\boldsymbol{\Phi}_{\mathrm{m}}, 76$ leading elements are selected. The reference map and the best representations with these two different bases are depicted in Figure 11b (left column) and show acceptable approximations.

The reference model in this experiment has similar connectivity patterns to those in the fifth group, i.e. $\mathbf{U}_{5}$. The reconstructed slowness map with the group-sparsity $\left(l_{1} / l_{2}\right.$-norm) regularization is able to clearly detect the straight left-to-right channels, with a less pronounced but clearly visible connectivity between them. The reconstruction coefficients are primarily selected from the consistent basis $\boldsymbol{\Phi}_{5}$. On the other hand, when $l_{1}$-norm regularization is used (Figure 11b, second row and second column), the reconstruction quality is not as good. More importantly, the reconstructed coefficients are distributed across several groups and do not identify any group as particularly relevant. The inversion with the mixed basis $\boldsymbol{\Phi}_{\mathrm{m}}$ fails to capture the correct connectivity pattern, which is consistent with the previous outcomes. 
Complex geologic patterns, such as meandering channels, present a parameterization challenge. While SVD-based parameterization is effective for multi-Gaussian variables or possibly straight channels, it is not suitable for preserving more complex meandering or curvilinear connectivity structures. This was demonstrated in Figure 6, where the TSVD basis elements for the meandering features do not contain these connectivity patterns. Figure 12a illustrates this issue with the tomographic reconstruction results, where a meandering channel is reconstructed using group-sparsity. Two meandering channels represent the reference slowness models, which are consistent with groups $\mathbf{U}_{1}$ or $\mathbf{U}_{2}$. The reconstruction results are shown in Figure 12a and 12b. A number of important observations from these two experiments are in order. First, the inadequacy of the TSVD parameterization in representing meandering features can be seen in Figure 12a. Although mainly the second group is selected, which consists of meandering features, the captured connectivity pattern in the center of the field is not crisp. This implies that the proposed group-sparsity formulation is affected by the limitations of the TSVD parameterization. While this limitation affects the performance of the group-sparsity formulation, it is caused by the parameterization and not the regularization method. Moreover, from the results in Figure 12, it can be seen that when the channels are parallel to the ray paths (Figure 12a) the solution can capture the existing channels. However, when two channels are perpendicular to the ray paths, e.g. in Figure 12b, the arrival time data from alone may not be sufficient to reveal the presence of two distinct channels.

\subsection{Aquifer Pumping Test in a Single Phase Flow}


We now shift our discussion to nonlinear problems, specifically focusing on single phase flow in groundwater aquifers. Three pumping-test experiments are considered, one using a 2D model (Case 3) and two with 3D models (Cases 4 and 5). In each case, a pumping well is placed at the center of the model domain and is extracting water with a constant flowrate of $0.0578 \frac{\mathrm{m}^{3}}{\mathrm{~s}}$ at the steady state aquifer conditions. The boundary conditions on the left and right of the domain are assigned constant pressures of $p_{1}=20 \mathrm{~m}$ and $p_{2}=10 \mathrm{~m}$, respectively, resulting in a background flow from left to right. The unknown parameters of interest are the spatial distribution of log-transmissivity and the relevant prior geologic scenario(s), which are to be inferred from observations of transmissivity and pressure head at scattered monitoring well locations surrounding the pumping well. The governing equations of the forward model are derived from Darcy's law and mass balance principle for a single phase flow in heterogeneous and saturated porous environment [25].

\section{Case 3: 2D Channel-Type Transmissivity}

The experimental setup for this case is depicted in Figure 13a. The aquifer has dimensions $1000 m \times 1000 m \times 10 m$ and is discretized into a domain with $100 \times 100 \times 1$ uniform grid cells. The reference transmissivity field is shown in Figure 13b (top left) and has low and high log-transmissivity values of -1.7047 (background) and -0.4037 (channel), respectively. The corresponding reference and initial pressure head fields are also shown in Figure 13b. The prior realization sets are the same as those depicted in Figure 5, with a total number of 10 geologic scenarios (groups). The TSVD parameterization is used to retain $80 \%$ of the energy for each group, resulting in $\boldsymbol{\Phi}_{\mathrm{g}} \in \mathrm{R}^{10000 \times 466}$. The best transmissivity map that can be constructed in the subspace defined by these bases is displayed in Figure 13b (top right). Data from 30 monitoring 
wells, randomly located around the pumping well (see Figure 13b, bottom left), are used for inversion. For this experiment, the reference model does not belong to any of the existing TIs; rather, it contains both intersecting and straight channel features, which can be reconstructed with the combination of geologic scenarios 7 and 9, that is using $\boldsymbol{\Phi}_{5}$ and $\boldsymbol{\Phi}_{9}$ bases.

Figure $13 \mathrm{c}$ depicts the reconstruction results for the log-transmissivity and pressure head fields using $\boldsymbol{\Phi}_{\mathrm{g}}$ and regularization with $l_{1} / l_{2}$-norm (top) and $l_{1}$-norm (bottom). The reconstruction solution with the group-sparsity regularization is clearly superior to that obtained by using the $l_{1}$-norm regularization. The stronger constraining power of group-sparsity clearly shows its advantage in this example as the limited scattered measurements have resulted in an underdetermined inverse problem. The group-sparse solution identifies significant coefficients from three groups, $\boldsymbol{\Phi}_{5}, \boldsymbol{\Phi}_{6}$ and $\boldsymbol{\Phi}_{9}$. While groups $\boldsymbol{\Phi}_{5}$ and $\boldsymbol{\Phi}_{9}$ are clearly relevant to the solutions, group $\boldsymbol{\Phi}_{6}$ also has channel elements along $\theta=135^{\circ}$ direction, which is consistent with the channel directionality in parts of the reference model. On the other hand, the inversion result with $l_{1}$-norm regularization selects several elements from various inconsistent groups and fails to reconstruct an acceptable connectivity pattern or identify an consistent prior model.

Figure 14 displays the behavior of the group-sparsity solution method as the gradientbased iterations proceed. The initial solution is picked from Group 1, which is incorrect. In early iterations, the group-sparsity algorithm uses the sensitivity information to activate (with very small coefficients) a number of potentially relevant groups, while reducing the contribution of Group 1. This leads to an overall decrease in the regularization term and a drop in the misfit term. As the iterations proceed, the data misfit term increases and the regularization term decreases in such a way that the overall objective function decreases with every iteration. The 
decrease in the objective function at later iteration is achieved by retaining and fine-tuning the final group(s) and removing the inconsistent ones.

An important issue that affects the final solution is related to well configuration. For a given number of wells, the solution of the inverse problem depends on the distribution and relative position of the wells. However, the pressure and flow data tend to provide more global information about the communication among the wells and the connectivity of the flow property fields, which may be used to discriminate against alternative scenarios.

\section{Case 4: 3D Multi-Gaussian Transmissivity}

In this experiment, an aquifer with dimensions $1000 \mathrm{~m} \times 1000 \mathrm{~m} \times 10 \mathrm{~m}$ is discretized into a $100 \times 100 \times 10$ uniform grid system. Figure 15a depicts the schematic of the domain and pumping test configuration. The reference log-transmissivity field has a multi-Gaussian structure and is shown in Figure 17b (top left) along with the best achievable reconstruction, as well as the corresponding pressure heads. The reference log-transmissivity map is generated using Variogram 1 in Table 1 with a $\theta=45^{\circ}$ direction of anisotropy. Three variogram models with parameters defined in Table 1 are used to represent the anisotropy ratios. For each variogram, four anisotropy directions, i.e., $\theta=0^{\circ}, 45^{\circ}, 90^{\circ}, 135^{\circ}$, are considered. As a result, 12 variogram models are used to represent the uncertainty in the prior geologic continuity scenario. Hence, 12 different prior distributions with various means and covariance matrices are suggested as alternative prior models. After stacking the bases generated by TSVD parameterization to retain $80 \%$ energy within each group, 1884 basis elements are included in $\boldsymbol{\Phi}_{\mathrm{g}}$. In general, to preserve $80 \%$ of the energy in each group, the prior groups with large-scale continuity patterns (Variogram 1) contain fewer basis elements than those with small-scale features (Variogram 3), 
see Figure 15c (right column). The observed data are noise-corrupted (with $2.5 \%$ noise) $\log$ transmissivity and pressure head at monitoring well locations.

The reconstruction results with group-sparsity and regular $l_{1}$-norm are shown in Figure 15c. The solution is initialized by giving equal weights to each group. At convergence, the group-sparsity regularization is able to eliminate inconsistent groups (Variograms 2 and 3) and retain mainly Group 2, corresponding to Variogram 1 . On the other hand, the solution of $l_{1}$-norm regularization contains several groups in the final solution. While the solution with $l_{1}$-norm recovers some of the trend that are similar to the spatial continuity features in the reference model, the overall connectivity patterns are not captured as accurately as they are with group sparsity. A comparison of the RMSE values from the two solutions also reveals the superiority of the results with group-sparsity. Finally, as in previous examples, no dominant prior model is identified with $l_{1}$-norm regularization, showing a completely different behavior than groupsparsity, in which distinct geologic scenarios with significant contribution to reproducing the data are selected.

\section{Case 5: 3D Channel Type Transmissivity}

In this example, an aquifer with dimensions $1000 m \times 1000 m \times 10 m$ is discretized into $100 \times$ $100 \times 9$ cells (see Figure 15a for schematic of the experiment). Each subsequent three layers in the formation are assumed to belong to the same geologic unit with distinct connectivity patterns. Three distinct TIs (Figure 5a) are used to represent the connectivity in the geologic units; however, it is not known a-priori which TI represent each of the three geologic units. With this setup, a total of 27 possible prior models are proposed and 500 realizations are generated to represent each prior model (see Figure 16 for two sample realizations). The TSVD 
parameterization basis for each prior is designed to preserve $80 \%$ of energy for each prior, leading to the combined 2411 basis elements, that is $\boldsymbol{\Phi}_{\mathrm{g}} \in \mathrm{R}^{90000 \times 2411}$.

The reference transmissivity field is depicted in Figure 17a. The first (layers 1-3), second (layers 4-6) and third (layers 7-9) geologic units consist of intersecting, straight and meandering channels, respectively. The detailed plot of the reference and initial transmissivity models and their corresponding pressure head fields are displayed in Figure 17b. The initial model is assumed to have meandering channel structure in all the layers. The reference model is similar in its connectivity structure to those belonging to group number 16 . The observations are collected from wells that are fully penetrated in the entire formations and are distributed in the field as shown in Figures 17a-b. As a result, there are $30 \times 9=270$ measurements of pressure head that can be used for prior model identification and model calibration.

Figure 18a depicts the reconstruction results for the transmissivity field at different iterations of the model calibration optimization. The connectivity type in layers 1-3 and 4-6 of the solution are consistent with those in the reference model. Layers 7-9 contain complex meandering channel features that are not amenable to PCA representation. Figure $18 \mathrm{~b}$ illustrates the $l_{2}$-norm of the coefficients for the 27 prior models in the iterations. The final result (the last row of Figure 18b) indicates that the most dominant coefficients belong to the group number 16; however, elements from groups 10,11, 12 and 22 also tend to contribute to the solution. Layers (1-3,4-6,7-9) of the prior models $10,11,12$ and 22 are generated from the training images $\left(\mathrm{TI}_{2}, \mathrm{TI}_{1}, \mathrm{TI}_{1}\right),\left(\mathrm{TI}_{2}, \mathrm{TI}_{1}, \mathrm{TI}_{2}\right),\left(\mathrm{TI}_{2}, \mathrm{TI}_{1}, \mathrm{TI}_{3}\right)$ and $\left(\mathrm{TI}_{3}, \mathrm{TI}_{2}, \mathrm{TI}_{1}\right)$, respectively. The basis functions corresponding to layers with intersecting and straight channels are better able to represent the common patterns in their group. On the hand, the basis functions for the meandering channels do not capture the meandering features properly and tend to contribute to 
representation of non-meandering channels. The pressure field corresponding to the solution is depicted in Figure 18c, and shows a significant improvement over the initial pressure field in Figure $17 b$.

\section{Conclusion}

Using flow-related data to constrain prior geologic scenarios is not typically performed in conventional subsurface flow model calibration. By exploiting the concept of group-sparsity in sparse reconstruction literature, we develop a systematic framework for simultaneously identifying geologic scenarios and estimating a calibrated model. The method generates several hundreds of realizations from each geologic scenario and uses them to construct a TSVD basis that can separately parameterize the models within each group. By combining the TSVD bases for each group a large hybrid basis is generated that is capable of compactly representing models from any group, or a model with features that cannot be found in a single group, but may be represented using multiple groups. However, since the groups are vastly different from each other only a small number of groups are needed to accurately represent a given model (or the inversion solution). This property translates into a group-sparse behavior that can be effectively induced using a mixed $l_{1} / l_{2}$-norm regularization term, which is minimized concurrently with a data mismatch objective function in an inverse problem.

The results in this paper clearly illustrate the properties of the group-sparse regularization and the advantages it has over regular $l_{1}$-norm based sparsity regularization. It is shown that promoting group-sparsity can be more powerful in constraining the solution of ill-posed modlel calibration problems. A particularly useful property of group-sparsity is its ability to discriminate against multiple prior geologic scenarios that account for the uncertainty in the knowledge of 
geologic continuity. Uncertainty in prior geologic scenario is an important topic that has not received its well-deserved attention in the literature. Traditionally, a given geologic scenarios, which is derived from limited available data, subjective interpretation, and imperfect modeling assumptions, is used to constrain the solution of model calibration problems. As such, the uncertainty in the geologic scenario is rarely studied in the context of model calibration. Interestingly, this source of uncertainty can have a detrimental impact on the quality of model calibration solutions and the related future predictions. Furthermore, the constraining power of flow data can be very informative in selecting plausible geologic scenarios and developing sound predictive models.

While in this study geologic continuity was represented with either TIs or variogram models, in general, uncertainty in geologic scenario can arise from various sources in subsurface flow model development workflows. Prior geologic scenarios can be derived from stochastic representation of process-based geologic models, or by alternative interpretations of the global connectivity structure by different geologists, or the combination of them. The main message of this paper is that, regardless of how the uncertainty in the geologic scenario is introduced and represented, the uncertainty in global geologic parameters can and should be incorporated in the model calibration formulation as it can have significant consequences in predicting flow and transport behavior of weakly-constrained and poorly-observed subsurface models. The proposed group-sparsity formulation in this paper offers an effective workflow to account for uncertainty in prior geologic scenarios. More importantly, it illustrates the feasibility of constraining uncertain geologic scenarios with flow related data, a novel concept that goes beyond the conventional subsurface model calibration formulations. 


\section{Appendix A: Group-Sparsity Inversion}

The regularized objective function of the group-sparsity formulation is expressed as

$$
\min _{\mathbf{v}} \quad J(\mathbf{v})=\left\|\mathbf{C}_{n}^{-1}\left(\mathbf{d}-\mathbf{g}\left(\mathbf{\Phi}_{g} \mathbf{v}\right)\right)\right\|_{2}^{2}+\alpha \sum_{i=1}^{p} \mu_{i}\left\|\mathbf{K}_{i} \mathbf{v}_{i}\right\|_{2}
$$

where the notations are discussed in the text. Using the Gauss-Newton method, at iteration $(n+1)$, the linearized version of the above function takes the form

$$
\begin{aligned}
\min _{\mathbf{v}^{(n+1)}} J\left(\mathbf{v}^{(n+1)}\right) & =\left\|\mathbf{C}_{n}^{-1}\left(\mathbf{d}-\mathbf{g}\left(\mathbf{\Phi} \mathbf{v}^{(n)}\right)-\mathbf{G}^{(n+1)}\left(\mathbf{v}^{(n+1)}-\mathbf{v}^{(n)}\right)\right)\right\|_{2}^{2} \\
& +\alpha \sum_{i=1}^{p} \mu_{i}\left(\sum_{j=1}^{s_{i}} k_{j j}^{i} v_{j}^{i(n+1)^{2}}\right)^{\frac{1}{2}}
\end{aligned}
$$

where $v_{j j}^{i}$ is the basis element $j$ in group $i$, and $k_{j j}^{i}$ is the $j^{\text {th }}$ diagonal element of the weight assigned to group $i$ (i.e., the $j^{\text {th }}$ element of $\left.\mathbf{K}_{i}^{T} \mathbf{K}_{i}\right) ; \mathbf{G}^{(n+1)}$ is the Jacobian matrix of $\mathbf{g}(\mathbf{v})$, which is calculated at $\mathbf{v}=\mathbf{v}^{(n)}$. With definitions $\Delta \mathbf{d}^{(n)}=\mathbf{d}-\mathbf{g}\left(\boldsymbol{\Phi}_{g} \mathbf{v}^{(n)}\right)+\mathbf{G}^{(n+1)} \mathbf{v}^{(n)}$ and $\overline{\mathbf{G}}^{(n+1)}=$ $\mathbf{C}_{n}^{-1} \mathbf{G}^{(n+1)},(\mathrm{A} 2)$ can be simplified to

$$
\min _{\mathbf{v}}(n+1) J\left(\mathbf{v}^{(n+1)}\right)=\left\|\mathbf{C}_{n}^{-1} \Delta \mathbf{d}^{(n)}-\overline{\mathbf{G}}^{(n+1)} \overline{\mathbf{v}}^{(n+1)}\right\|_{2}^{2}+\alpha \sum_{i=1}^{p} \mu_{i}\left(\sum_{j=1}^{s_{i}} k_{j j}^{i} v_{j}^{i(n+1)^{2}}\right)^{\frac{1}{2}}
$$

The derivative of the regularization term with respect to $v_{j}^{i(n+1)}$ can be approximated as

$$
\frac{\partial J_{2}^{(n+1)}}{\partial v_{i j}^{(n+1)}}=\frac{\mu_{i} k_{j j}^{i} v_{j}^{i(n+1)}}{\left(\sum_{j=1}^{n_{i}} k_{j j}^{i} v_{j}^{i(n+1)^{2}}\right)^{\frac{1}{2}}} \approx \frac{\mu_{i} k_{j j}^{i} v_{j}^{i(n+1)}}{\left(\sum_{j=1}^{n_{i}} k_{j j}^{i} v_{j}^{i(n)^{2}}+\epsilon_{i}^{(n+1)}\right)^{\frac{1}{2}}}
$$

where $\epsilon_{i}^{(n+1)}$ is a small positive number that is used to avoid zero denominators. Note that $v_{j}^{i(n+1)}$ in the denominator is approximated with $v_{j}^{i(n)}$. Choosing $\epsilon$ such that $0<\epsilon_{i}^{(n+1)}<\epsilon_{i}^{(n)}$ and $\lim _{n \rightarrow \infty} \epsilon_{i}^{(n)}=0$, it can be shown that this approximation does not change the solution of the original minimization problem. The iterative solution of Eq. (A3) can now be derived as

$$
\left(\overline{\mathbf{G}}^{(n+1)^{T}} \overline{\mathbf{G}}^{(n+1)}+\alpha \mathbf{\Lambda}^{(n+1)}\right) \overline{\mathbf{v}}^{(n+1)}=\overline{\mathbf{G}}^{\prime} \mathbf{C}_{n}^{-1} \Delta \mathbf{d}^{(n)}
$$


where $\boldsymbol{\Lambda}^{(n+1)}$ is a diagonal matrix with diagonal entries $\frac{\mu_{i} k_{j j}^{i}}{\left(n_{j} k_{i} i(n)^{2}\right.}$. In this work, the Jacobian matrix is derived from an adjoint model that is implemented in the forward simulation.

\section{Appendix B: Pseudo Code for the Entire Process}

The overall procedure for generating prior realizations, performing inversion and model selection is presented below:

1. Use the proposed $p$ prior conceptual models and a geostatistical simulation technique to generate sample $N$ realizations for each prior and collect the realizations from each prior scenario in a separate matrix, i.e., $\mathbf{U}_{1}=\left[\begin{array}{l}\mathbf{u}_{11} \\ \mathbf{u}_{12} \ldots \mathbf{u}_{1 N}\end{array}\right], \ldots, \mathbf{U}_{p}=\left[\mathbf{u}_{p 1} \mathbf{u}_{p 2} \ldots \mathbf{u}_{p N}\right]$,

2. Apply SVD on $\mathbf{U}_{1}, \mathbf{U}_{2}, \ldots, \mathbf{U}_{p}$, to construct the TSVD bases $\boldsymbol{\Phi}_{1}, \ldots, \boldsymbol{\Phi}_{p}$ for different scenario, and form $\boldsymbol{\Phi}_{\mathrm{g}}=\left[\boldsymbol{\Phi}_{1}, \ldots, \boldsymbol{\Phi}_{p}\right]$.

3. Use $\mathbf{U}_{1}, \ldots, \mathbf{U}_{p}$ to train the weights $\mathbf{K}_{1}, \ldots, \mathbf{K}_{p}$ (see Eq. (8)).

4. Perform group-sparse model calibration following the formulation in Eq. (8) and Appendix A with $\mathbf{d}=\mathbf{g}(\mathbf{u})$ representing the relation between the flow data and parameters.

5. Identify the active groups $\mathbf{v}_{1}, \ldots, \mathbf{v}_{S \ll p}$ in representing the solution based on the raking of their $l_{2}$-norm (these are the dominant geologic scenarios).

6. After analysis of the selected groups, if necessary, propose revised/refined prior scenarios and repeat steps 1-5.

7. If a single geologic scenario is identified, standard model calibration and uncertainty quantification with the selected prior scenario can be performed. 


\section{Acknowledgement}

The work in this paper is supported partially by the Department of Energy. All data and digital content in this manuscript can be accessed by contacting the corresponding author at behnam.jafarpour@usc.edu. 


\section{References}

[1] Ahmed N, Natarajan T, Rao KR. Discrete cosine transform. Computers, IEEE Transactions on $1974 ; 100(1), 90-93$.

[2] Akaike H. A new look at the statistical model identification. Automatic Control, IEEE Transactions on. 1974 Dec;19(6):716-23.

[3] Aster RC, Borchers B, Thurber CH. Parameter estimation and inverse problems. Academic Press, 2011.

[4] Bach F, Jenatton R, Mairal J, Obozinski G. Structured sparsity through convex optimization. Statistical Science 2012; 27(4), 450-468.

[5] Backus G., Gilbert F. (1968), The resolving power of gross earth data. Geophysical Journal of the Royal Astronomical Society, 16, 169-205.

[6] Bear J. Dynamics of fluids in porous media.1972, New York: American Elsevier Pub. Co.; 764p. ISBN 9780444001146.

[7] Bregman N. D., Bailey R.C., Chapman C.H. (1989), Crosshole seismic tomography, Geophysics, v. 54, p. 200-215, published online February 1, 1989, doi:10.1190/1.1442644.

[8] Bissell R. Calculating optimal parameters for history matching. Paper presented at 4th European Conference on the Mathematics of Oil Recovery 1994.

[9] Caers J. Geostatistical history matching under training-image based geological model constraints. In SPE annual technical conference and exhibition, January 2002. Society of Petroleum Engineers.

[10] Caers J, Zhang T. Multiple-point geostatistics: a quantitative vehicle for integrating geologic analogs into multiple reservoir models. AAPG Special Volumes 2004; 383-394. 
[11] Candes EJ, Romberg JK, Tao T. Stable signal recovery from incomplete and inaccurate measurements. Communications on pure and applied mathematics 2006; 59(8), 1207-1223.

[12] Carrera J, Alcolea A, Medina A, Hidalgo J, Slooten LJ. Inverse problem in hydrogeology. Hydrogeol J 2005;13(1):206-22. http://dx.doi.org/10.1007/s10040-004-0404-7.

[13] Carrera J, Neuman SP. Estimation of aquifer parameters under transient and steady state conditions: 1. Maximum likelihood method incorporating prior information. Water Resour Res 1986a; 22(2):199-210. http://dx.doi.org/10.1029/WR022i002p00199.

[14] Carrera J, Neuman SP. Estimation of aquifer parameters under transient and steady state conditions: 2. Uniqueness, stability, and solution algorithms. Water Resour Res 1986b; 22(2):211-27. http://dx.doi.org/10.1029/WR022i002p00211.

[15] Coats KH, Dempsey JR, Henderson JH. A new technique for determining reservoir description from field performance data. Society of Petroleum Engineers Journal $1970 ; 10(01), 66-74$.

[16] Cressie N, Hawkins DM. Robust estimation of the variogram: I.Journal of the International Association for Mathematical Geology 1980; 12(2), 115-125.

[17] De Marsily G, Delhomme JP, Coudrain-Ribstein A, Lavenue AM. Four decades of inverse problems in hydrogeology. In: Zhang D, Winter CL, editors. Theory, modeling, and field investigation in hydrogeology: a special volume in honor of Shlomo P. Neumans 60th birthday, geological society of America special paper 348, 2000; Boulder, Colorado; p. 1-17. http://dx.doi.org/10.1130/0-8137-2348-5.1.

[18] De Marsily G, Lavedau G, Boucher M, Fasanino G. Interpretation of interference tests in a well field using geostatistical techniques to fit the permeability distribution in a reservoir model. In: Verly G, David M, Journel AG, Marechal A, editors. Geostatistics for natural 
resources characterization. Hingham, MA: D. Reidel; 1984. p. 831-49. http://dx.doi.org/10.1007/978-94-009-3701-7_16.

[19] Deutsch CV, Journel AG. GSLIB: Geostatistical software library and user's guide: Oxford Univ. Press 1992; New York, 340.

[20] Donoho DL. Compressed sensing, Information Theory, IEEE Transactions on 2006; 52(4), $1289-1306$.

[21] Efron B, Hastie T, Johnstone I, Tibshirani R. Least angle regression. The Annals of statistics 2004; 32(2), 407-499.

[22] Eldar YC, Kuppinger P, Bölcskei H. Block-sparse signals: Uncertainty relations and efficient recovery. Signal Processing, IEEE Transactions on 2010; 58(6), 3042-3054.

[23] Emsellem Y, De Marsily G. An automatic solution for the inverse problem. Water Resources Research 1971; 7(5), 1264-1283.

[24] Foglia L, Mehl SW, Hill MC, Burlando P. Evaluating model structure adequacy: The case of the Maggia Valley groundwater system, southern Switzerland. Water Resources Research. 2013 Jan 1;49(1):260-82.

[25] Franssen, H., A. Alcolea, M. Riva, M. Bakr, N. van der Wiel, F. Stauffer, and A. Guadagnini (2009), A comparison of seven methods for the inverse modeling of groundwater flow. Application to the characterization of well catchments., Advances in Water Resources, $32,851-872$.

[26] Gavalas GR, Shah PC, Seinfeld JH. Reservoir history matching by Bayesian estimation. Society of Petroleum Engineers Journal 1976; 16(06), 337-350. 
[27] Golmohammadi A, Khaninezhad MR, Jafarpour B. Group-Sparsity Regularization for IllPosed Subsurface Flow Inverse Problems. Water Resources Research, (2015, in press), DOI: 10.1002/2014WR016430.

[28] Golub GH, Reinsch C. Singular value decomposition and least squares solutions. Numerische mathematik 1970; 14(5), 403-420.

[29] Golub GH, Hansen PC, O’Leary DP. Tikhonov regularization and total least squares, SIAM Journal on Matrix Analysis and Applications 1999; 21(1), 185-194.

[30] Golub GH, Heath M, Wahba G. Generalized cross-validation as a method for choosing a good ridge parameter. Technometrics 1979; 21(2), 215-223.

[31] Hansen PC. Analysis of discrete ill-posed problems by means of the L-curve. SIAM review 1992; 34(4), 561-580.

[32] Hansen PC. Rank-deficient and discrete ill-posed problems: numerical aspects of linear inversion. SIAM 1998; DOI: http://dx.doi.org/10.1137/1.9780898719697.

[33] Hill MC, Tiedeman CR. Effective groundwater model calibration: with analysis of data, sensitivities, predictions, and uncertainty. John Wiley \& Sons, 2006.

[34] Huang J, Zhang T. The benefit of group sparsity. The Annals of Statistics 2010; 38(4), 1978-2004.

[35] Hurvich CM, Tsai CL. Regression and time series model selection in small samples. Biometrika. 1989 Jun 1;76(2):297-307.

[36] Jacquard P, Jain C. Permeability distribution from field pressure data, Soc. Pet. Eng. J. $1965 ; 5(4), 281-294$.

[37] Jafarpour B, McLaughlin DB. Reservoir characterization with the discrete cosine transform, SPE Journal 2009; 14(01), 182-201. 
[38] Jafarpour B, Goyal VK, McLaughlin DB, Freeman WT. Transform-domain sparsity regularization for inverse problems in geosciences. Geophysics 2009; 74(5), R69-R83.

[39] Jafarpour B, Goyal VK, McLaughlin DB, Freeman WT. Compressed history matching: Exploiting transform-domain sparsity for regularization of nonlinear dynamic data integration problems, Math. Geosci. 2010; 42(1), 1-27.

[40] Jafarpour B. Wavelet reconstruction of geologic facies from nonlinear dynamic flow measurements, IEEE Trans. Geosci. RemoteSens. 2011; 49(5), 1520-1535.

[41] Jafarpour B, Khodabakhshi M. A probability conditioning method (PCM) for nonlinear flow data integration into multipoint statistical facies simulation. Math Geosci 2011; 18748961;43(2):133-64. http://dx.doi.org/10.1007/s11004-011-9316-y.

[42] Jafarpour B, Tarrahi M. Assessing the performance of the ensemble Kalman filter for subsurface flow data integration under variogram uncertainty, Water Resources Research 2011; Vol. 47, W05537, 26 PP, DOI: 10.1029/2010WR009090.

[43] Jahns HO. A rapid method for obtaining a two-dimensional reservoir description from well pressure response data, Soc. Pet. Eng. J. 1966; 6(4), 315-327.

[44] Jolliffe I. Principal component analysis. John Wiley \& Sons, Ltd, 2002.

[45] Karhunen K. Über lineare Methoden in der Wahrscheinlichkeitsrechnung 1947; (Vol. 37). Universitat Helsinki.

[46] Kashyap RL. Optimal choice of AR and MA parts in autoregressive moving average models. Pattern Analysis and Machine Intelligence, IEEE Transactions on. 1982 Mar(2):99104.

[47] Khaninezhad MR, Jafarpour B, Li L. Sparse geologic dictionaries for subsurface flow model calibration: Part I. Inversion formulation. Advances in Water Resources 2012a; 39, 106-121. 
[48] Khaninezhad MM, Jafarpour B, Li L. Sparse geologic dictionaries for subsurface flow model calibration: Part II. Robustness to uncertainty. Advances in Water Resources $2012 b ; 39,122-136$.

[49] Khaninezhad MR, Jafarpour B. Prior model identification during subsurface flow data integration with adaptive sparse representation techniques. Computational Geosciences 2014; Vol. 18 (1), PP 3-16.

[50] Khodabakhshi M, Jafarpour B. A Bayesian mixture modeling approach for flowconditioned multiple-point statistical facies simulation from uncertain training images. Water Resources Research 2013; Vol. 49 (1), PP 328-342, DOI:10.1029/2011WR010787.

[51] Kitanidis PK. Parameter uncertainty in estimation of spatial functions: Bayesian analysis. Water Resources Research 1986; Vol. 22, (4), 499-507.

[52] Kitanidis PK, Vomvoris EG. A geostatistical approach to the inverse problem in groundwater modeling (steady state) and one-dimensional simulations. Water Resour Res 1983;19(3):677-90. http://dx.doi.org/10.1029/WR019i003p00677.

[53] Koltermann CE, Gorelick SM. Heterogeneity in sedimentary deposits: a review of structureimitating, process-imitating, and descriptive approaches. Water Resour Res 1996; 32(9):2617-58. 10.1029/96WR00025.

[54] LaVenue AM, RamaRao BS, De Marsily G, Marietta MG. Pilot point methodology for automated calibration of an ensemble of conditionally simulated transmissivity fields: 2 . Application. Water Resour Res, 0043-1397 1995;31(3):495-516. http://dx.doi.org/10.1029/94WR02259.

[55] Li L, Jafarpour B. Effective solution of nonlinear subsurface flow inverse problems in sparse bases. Inverse Problems 2010; 26(10), 105-016 (24pp). 
[56] Loeve M. Probability theory, vol. ii. Graduate texts in mathematics 1978; 46, 0-387.

[57] Lu P, Horne RN. A multiresolution approach to reservoir parameter estimation using wavelet analysis, paper presented SPE annual technical conference 2000, Soc. of Pet. Eng.

[58] Mallat S. A wavelet tour of signal processing: the sparse way; Third Ed, Academic Press 2008, Elsevier, Burlington, MA.

[59] McLaughlin D, Townley LR. A reassessment of the groundwater inverse problem. Water Resour Res 1996;32(5):1131-61. http://dx.doi.org/10.1029/96WR00160.

[60] Moore C, Doherty J. Role of the calibration process in reducing model predictive error. Water Resour Res 2005; 41(5). http://dx.doi.org/10.1029/2004WR003501.

[61] Moore C, Doherty J. The cost of uniqueness in groundwater model calibration. Adv Water Resour 2006;29(4):605-23. http://dx.doi.org/10.1016/j.advwatres.2005.07.003.

[62] Moore C, Wohling T, Doherty J. Efficient regularization and uncertainty analysis using a global optimization methodology, Water Resources Research 2010; 46(8), W08527. DOI: 10.1029/2009WR008627.

[63] Obozinski G, Jacob L, Vert JP. Group lasso with overlaps: the latent group lasso approach, 2011; arXiv preprint arXiv:1110.0413.

[64] Oliver DS, Chen Y. Recent progress on reservoir history matching: a review. Comput Geosci 2010;15(1):185-221. http://dx.doi.org/10.1007/s10596-010-9194-2.

[65] Oliver DS, Reynolds AC, Liu N. Inverse theory for petroleum reservoir characterization and history matching. Cambridge University Press 2008.

[66] Peyr'e G, Fadili J. Group sparsity with overlapping partition functions, Proc. EUSIPCO 2011; pp. 303-307. 
[67] RamaRao B, LaVenue A, De Marsily G, Marietta M. Pilot point methodology for automated calibration of an ensemble of conditionally simulated transmissivity fields: 1 . Theory and computational experiments. Water Resour Res 1995;31(3):475-93. http://dx.doi.org/10.1029/94WR02258.

[68] Rao KR, Yip P. Discrete cosine transform: algorithms, advantages, applications. Academic press, 2014.

[69] Refsgaard JC. Parameterisation, calibration and validation of distributed hydrological models. Journal of Hydrology 1997; 198(1-4), 69-97.

[70] Refsgaard JC, Christensen S, Sonnenborg TO, Seifert D, Højberg AL, Troldborg L. Review of strategies for handling geological uncertainty in groundwater flow and transport modeling. Advances in Water Resources 2012; 36, 36-50.

[71] Riva M, Panzeri M, Guadagnini A, Neuman SP. Role of model selection criteria in geostatistical inverse estimation of statistical data-and model-parameters. Water Resour Res 2011;47(7). http://dx.doi.org/10.1029/2011WR010480.

[72] Rudin LI, Osher S, Fatemi E. Nonlinear total variation based noise removal algorithms. Physica D: Nonlinear Phenomena 1992; 60(1), 259-268.

[73] Scales JA, Docherty P, Gersztenkorn A. Regularisation of nonlinear inverse problems: imaging the near-surface weathering layer. Inverse Problems 1990; 6(1), 115.

[74] Stojnic M, Parvaresh F, Hassibi B. On the reconstruction of block-sparse signals with an optimal number of measurements. Signal Processing, IEEE Transactions on 2009; 57(8), 3075-3085.

[75] Strebelle S. Conditional simulation of complex geological structures using multiple-point statistics. Mathematical Geology 2002; 34(1), 1-21. 
[76] Schwarz G. Estimating the dimension of a model. The annals of statistics. 1978;6(2):461-4.

[77] Tarantola A. Inverse problem theory and methods for model parameter estimation. SIAM, 2005.

[78] Tikhonov A. Solution of incorrectly formulated problems and the regularization method. In Soviet Math. Dokl. 1963; Vol. 5, pp. 1035-1038.

[79] Tsai FTC, Yeh WWG. Characterization and identification of aquifer heterogeneity with generalized parameterization and Bayesian estimation. Water Resources Research 2004; 40(10). DOI: 10.1029/2003WR002893.

[80] Wold S, Esbensen K, Geladi P. Principal component analysis. Chemometrics and intelligent laboratory systems 1987; 2(1), 37-52.

[81] Ye M, Meyer PD, Neuman SP. On model selection criteria in multimodel analysis. Water Resources Research. 2008 Mar 1;44(3).

[82] Yeh WWG. Review of parameter identification procedures in groundwater hydrology: The inverse problem. Water Resources Research 1986;22(2), 95-108.

[83] Zhou H, Gómez-Hernández JJ, Li L. Inverse methods in hydrogeology: Evolution and recent trends, Advances in Water Resources 2014, Volume 63, Pages 22-37, ISSN 03091708, http://dx.doi.org/10.1016/j.advwatres.2013.10.014. 


\section{Tables}

Table1. Variogram parameters for Case 4 in Section 3.2.

\begin{tabular}{|c|c|c|c|}
\hline & $\boldsymbol{\gamma}_{\mathbf{1}}$ & $\boldsymbol{\gamma}_{\mathbf{2}}$ & $\boldsymbol{\gamma}_{\mathbf{3}}$ \\
\hline $\boldsymbol{a}_{\boldsymbol{m a x}}$ & 800 & 400 & 200 \\
\hline $\boldsymbol{a}_{\text {med }}$ & 400 & 100 & 50 \\
\hline $\boldsymbol{a}_{\text {min }}$ & 400 & 100 & 50 \\
\hline
\end{tabular}




\section{Figures}

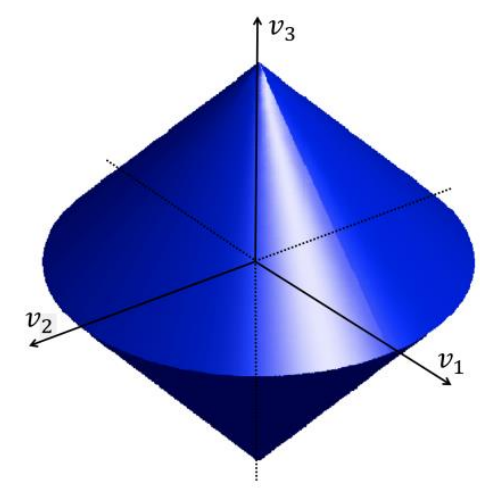

(a)

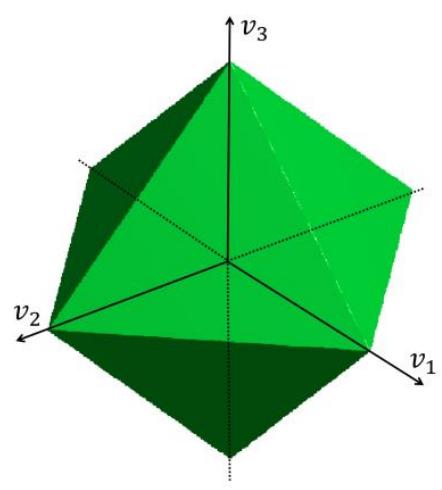

(b)

Figure 1. Comparison between the balls of group-sparsity and regular sparsity inducing norms in three dimensions, that is for $\mathbf{v}=\left[v_{1} v_{2} v_{3}\right]$ : (a) $l_{1} / l_{2}$-norm using two groups $\left[v_{1}, v_{2}\right]$ and $\left[v_{3}\right]$, resulting in $\left(v_{1}^{2}+v_{2}^{2}\right)^{\frac{1}{2}}+\left|v_{3}\right|$ and (b) $l_{1}$-norm, i.e., $\left|v_{1}\right|+\left|v_{2}\right|+\left|v_{3}\right|$ (Figure is borrowed from [Bach et al., 2012]). 
(a)

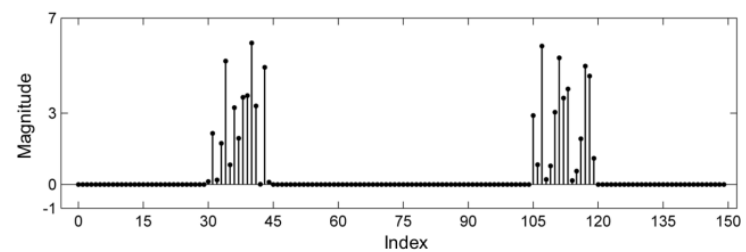

Signal Length : 150

Sparsity : $30(2 \times 15)$

N. of Groups : 10

Bases per Group : 15
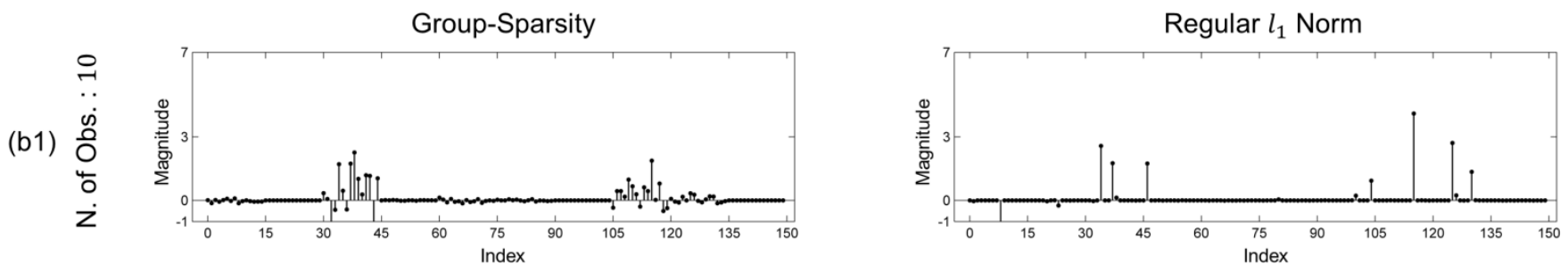

(b2)
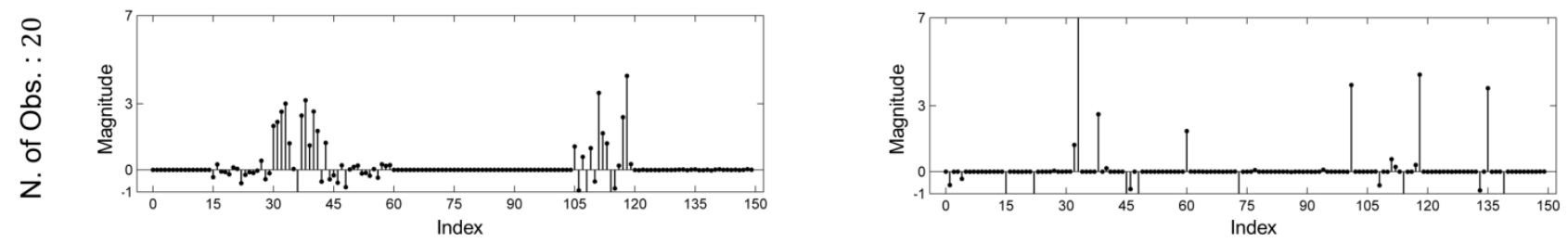

(b3)
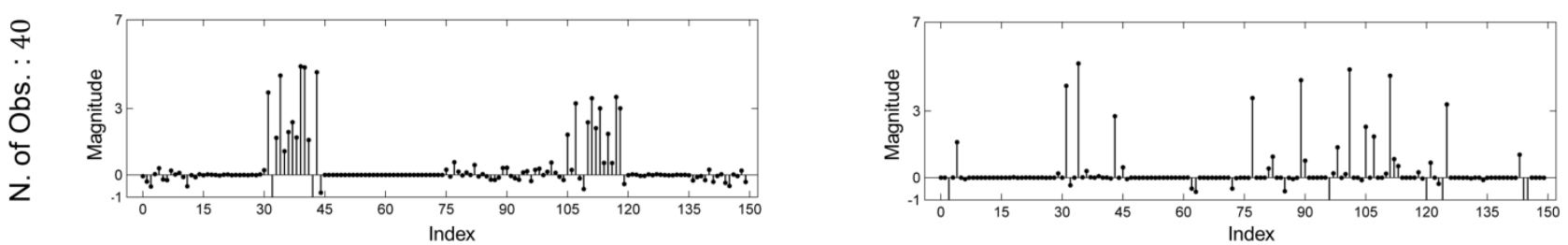

(b4)
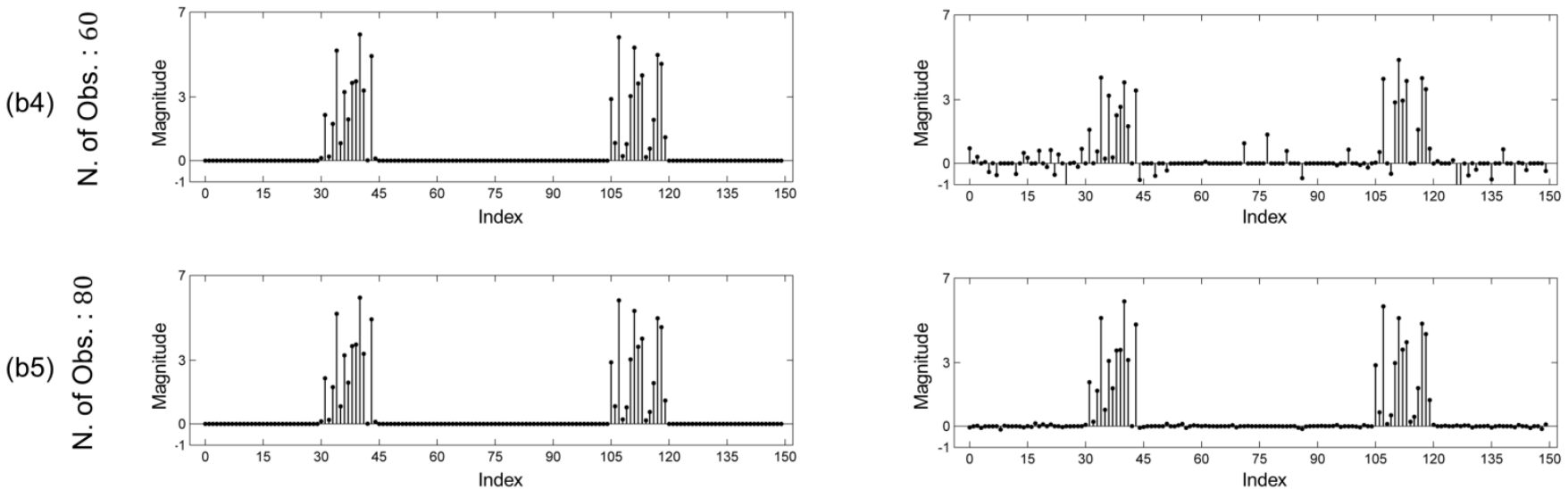

Figure 2. Comparison between reconstruction results with group-sparsity and $l_{1}$-norm regularization for different number of observations: (a) original group sparse signal; (b1)-(b5) reconstruction results for group-sparsity and regular $l_{1}$-norm sparsity inducing regularization for 10, 20, 40, 60 and 80 observations, respectively. 
(a) Smaller Groups

(a1)

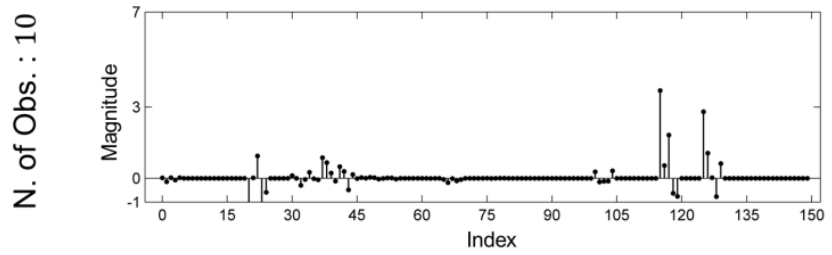

(a2)

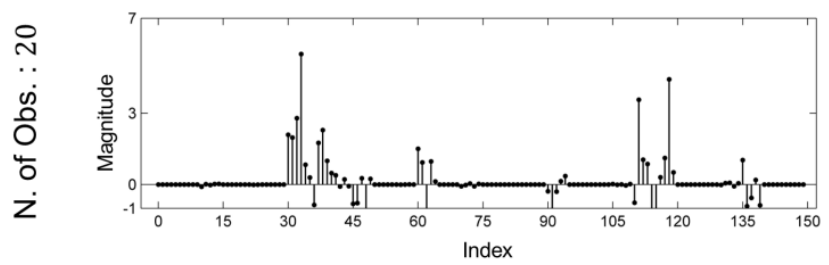

(a3)

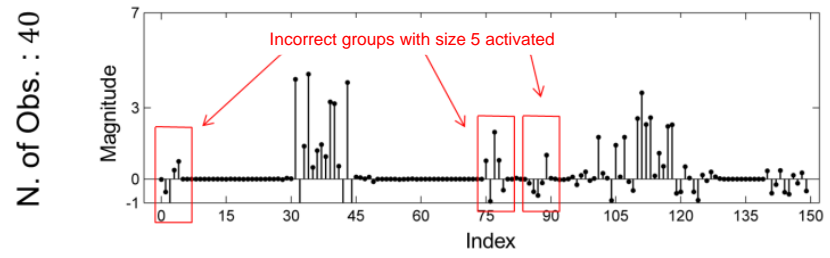

(a4)

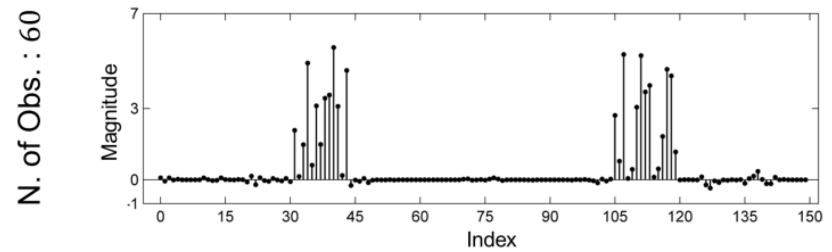

(a5)

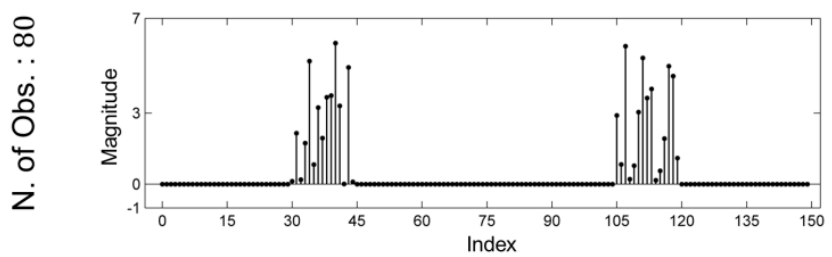

(b) Incorrect Grouping

(b1)

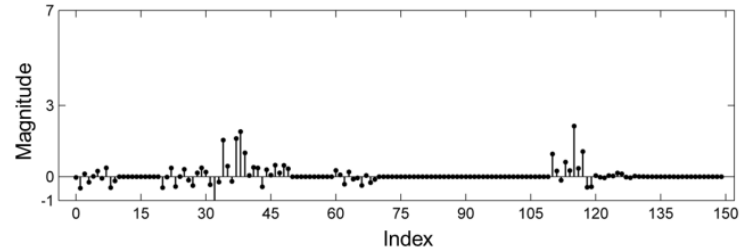

(b2)

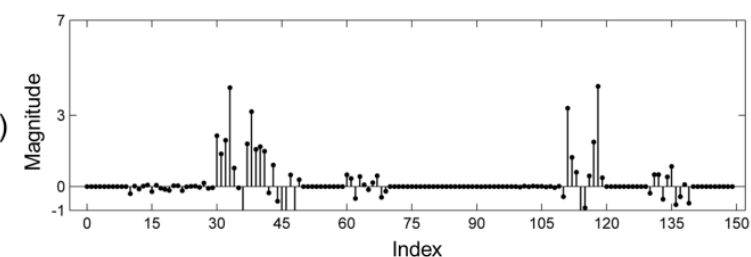

(b3)

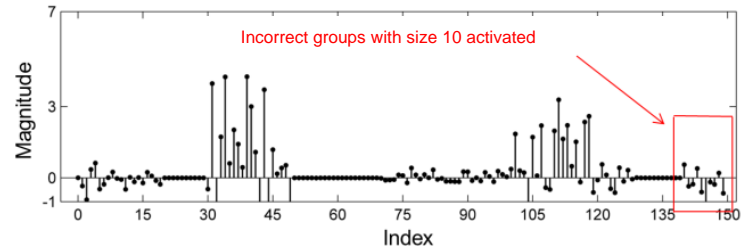

(b4)

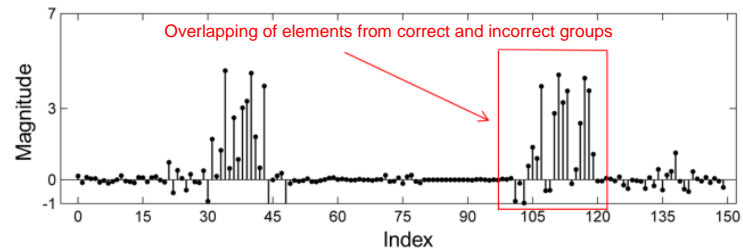

(b5)

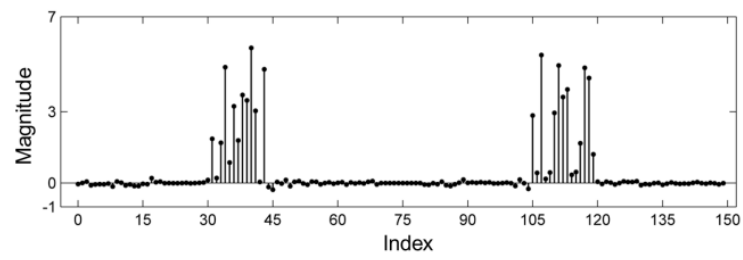

Figure 3. Reconstructing results for the group-sparse signal in Figure 2 for: (a) groups with size 5 , buy splitting the original groups of size 10 (overlapping case); (b) groups with size 10 with no overlapping. In the latter case, the grouping structure is inconsistent with the structure in the reference model. 
(a) Reconstruction for Various Values of $\alpha$
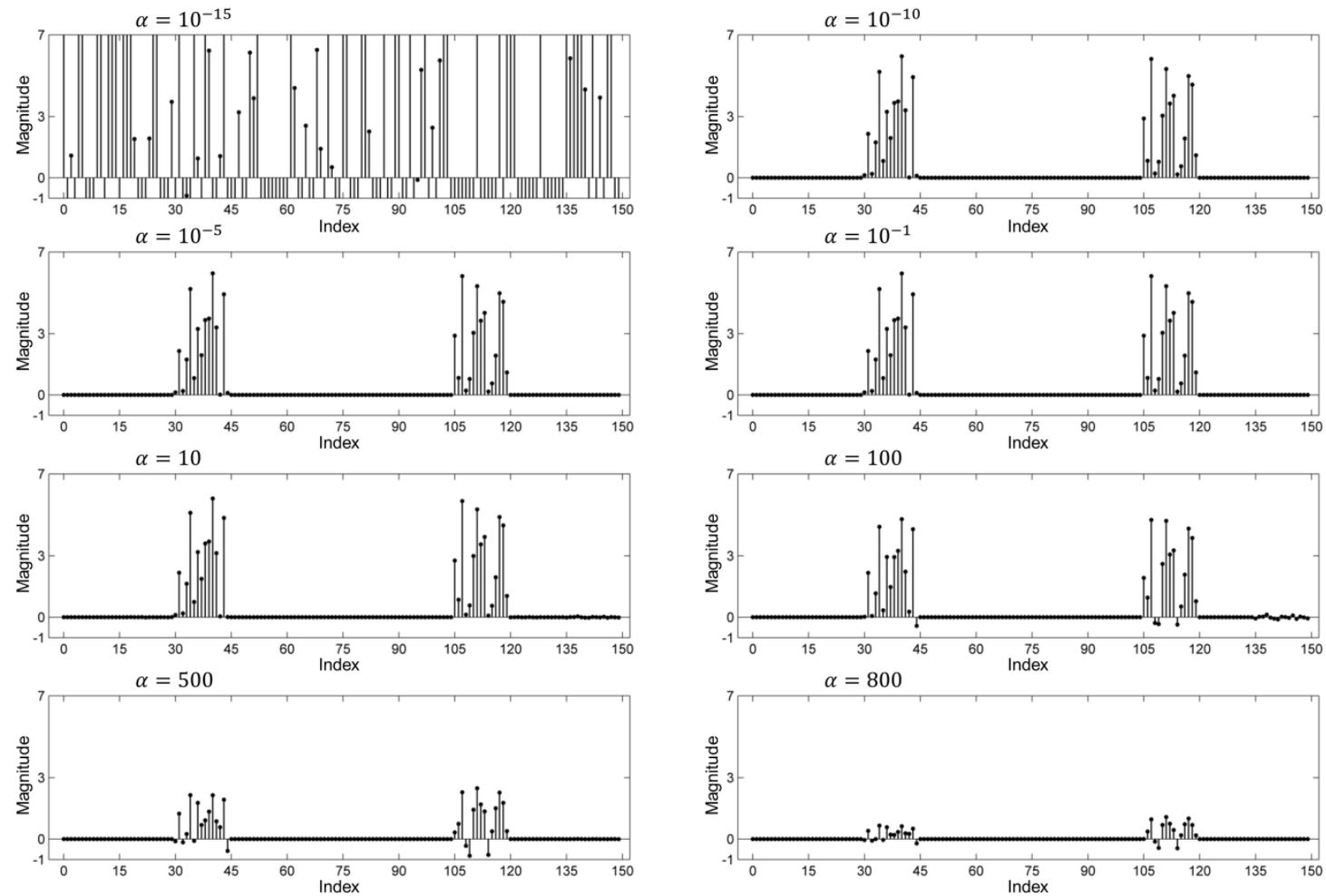

(b) Data Match for Various Regularization Parameters

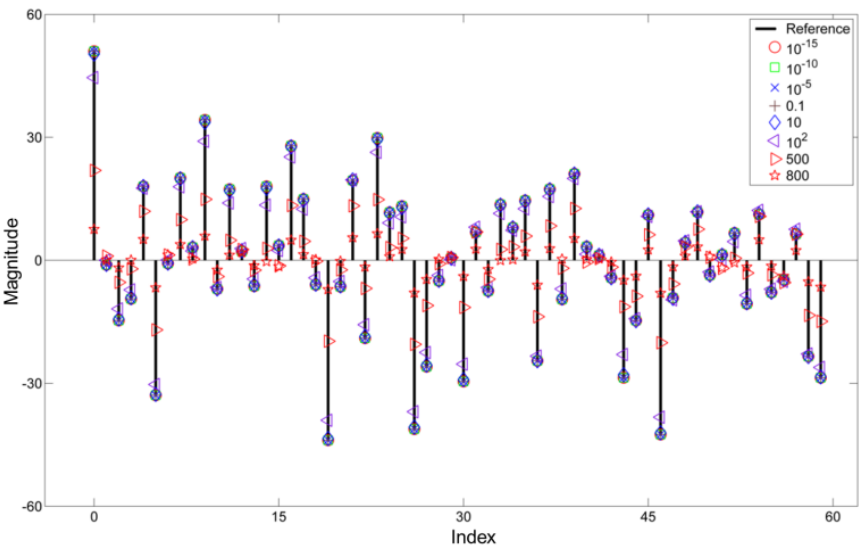

Figure 4. (a) Reconstruction results for the case with 60 measurements (Figure 2(b4)) with different regularization parameters; (b) data match for reconstructed signal with the corresponding regularization parameter in (a). 

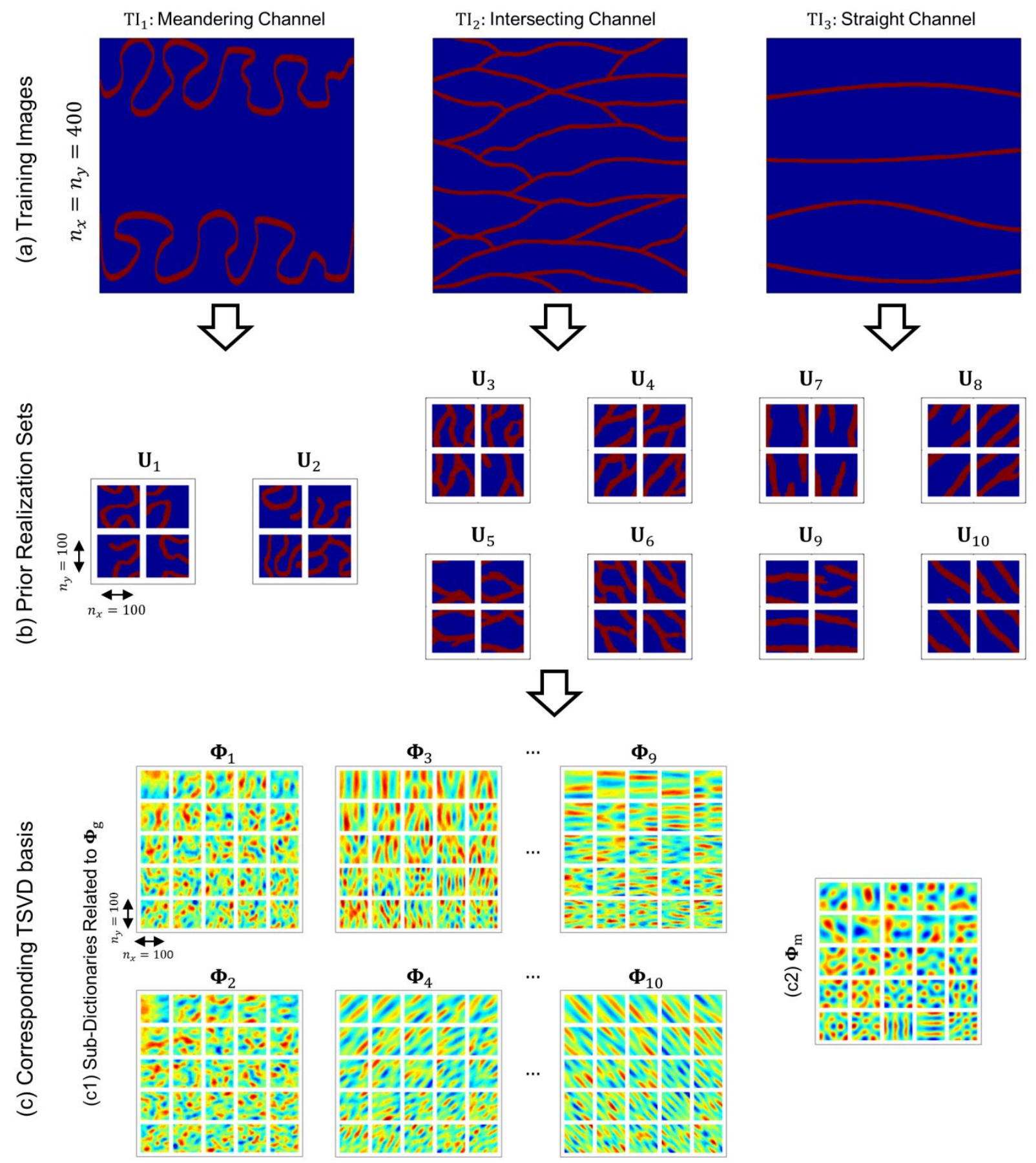

Figure 5. (a) Three TIs with meandering (left), intersecting (middle), and straight (right) channel features; (b) four sample realizations simulated from each TI. The TI with meandering channel is considered with two rotation angles $\left(\theta=0^{\circ}\right.$ and $\left.\theta=90^{\circ}\right)$ while the other two TIs each lead to four alternative scenario with rotation angles $\theta=0^{\circ}, 45^{\circ}, 90^{\circ}, 135^{\circ}$; (c) Sample TSVD basis elements corresponding to different geologic scenarios, i.e., $\boldsymbol{\Phi}_{\mathrm{g}}=\left[\boldsymbol{\Phi}_{1} \boldsymbol{\Phi}_{2} \ldots \boldsymbol{\Phi}_{10}\right]$ and the TSVD basis obtained after combining all prior realizations, i.e., $\boldsymbol{\Phi}_{\mathrm{m}}$. 

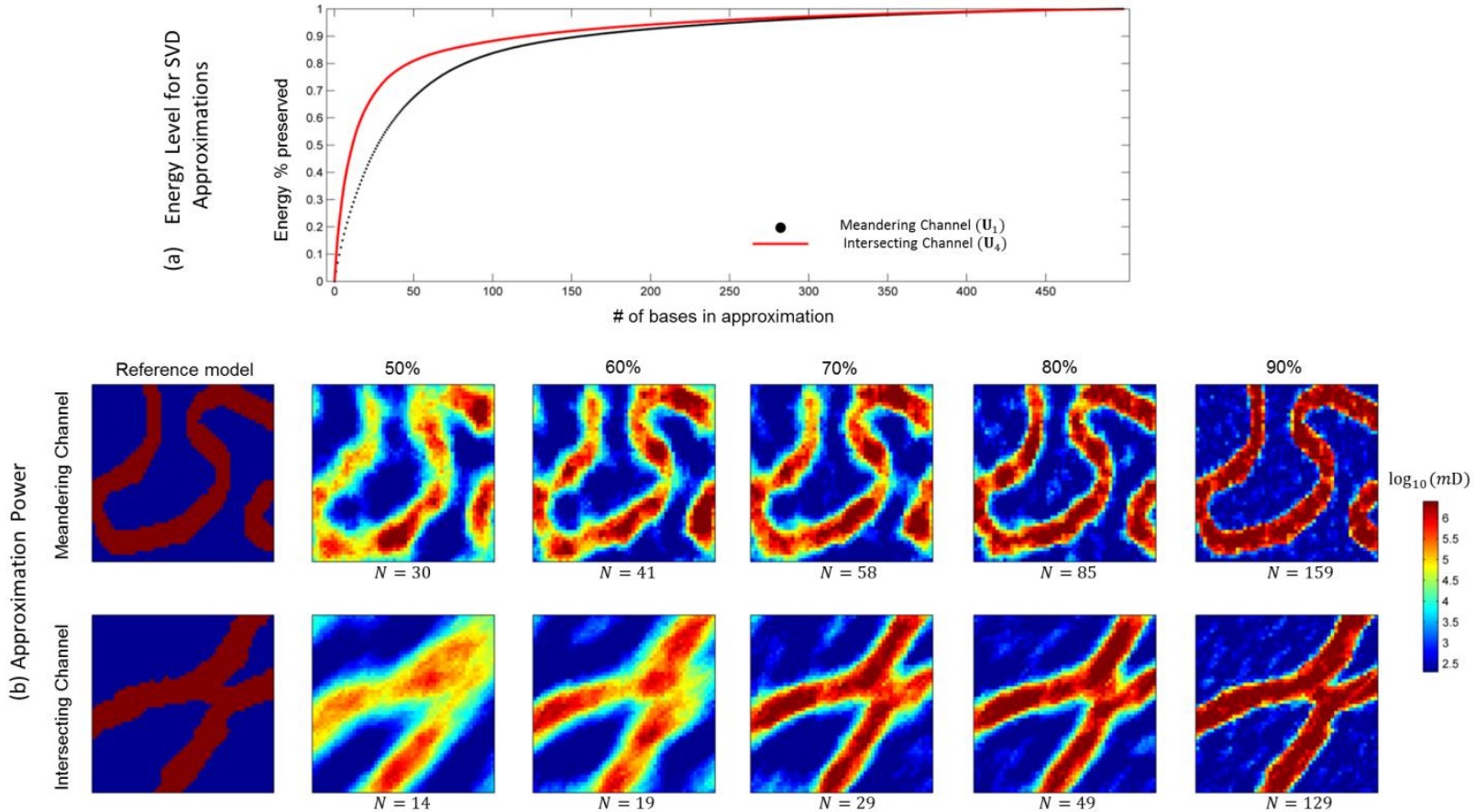

$\%$ : percentage of preserved energy

$N$ : number of bases at corresponding energy level

Figure 6. (a) Eigen-spectrum of the prior models for groups $\mathbf{U}_{1}$ (meandering channel) and $\mathbf{U}_{4}$ ((intersecting channel); (b) Corresponding TSVD approximation quality for different level of energy. 
Compression on Multiple Groups and Comparison of Misfit and $l_{2}$-Norms

Approximations
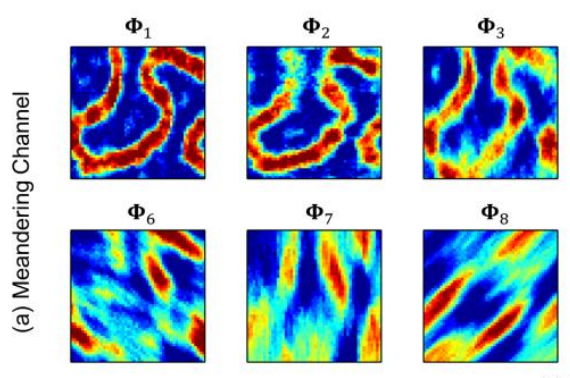

$n_{x}=n_{y}=100$
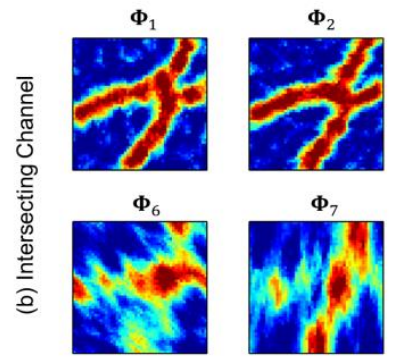

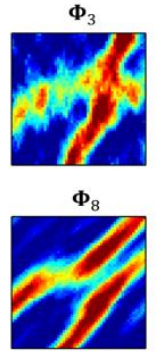

$n_{x}=n_{y}=100$

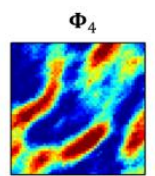

$\Phi_{9}$

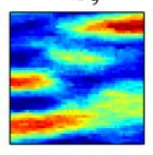

$\Phi_{4}$

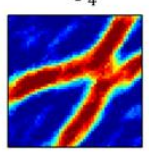

$\Phi_{9}$

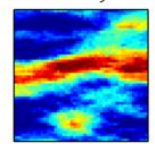

$x$

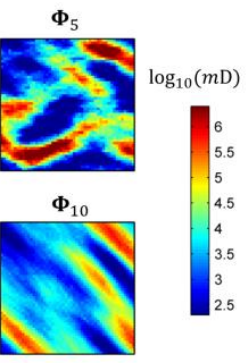

$\boldsymbol{\Phi}_{5}$

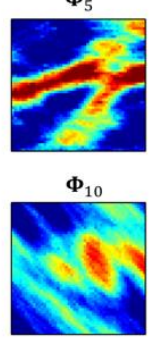

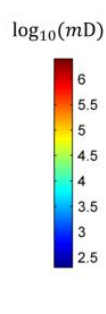

Misfit and $l_{2}$-norm
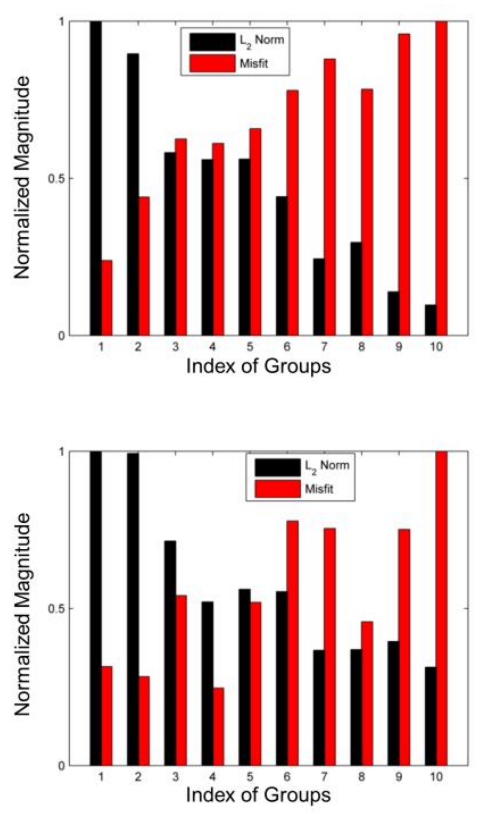

Figure 7. Approximation of sample models with TSVD bases from different groups: (a) meandering channel example; (b) intersecting channel example; (c) bar plots of the normalized data misfit (RMSE) and the $l_{2}$-norm of the best approximation coefficients. 
Prior Model Identification based on Compression on $\boldsymbol{\Phi}_{\mathrm{g}}$ and Using $l_{1} / l_{2}$ Norm Regularization

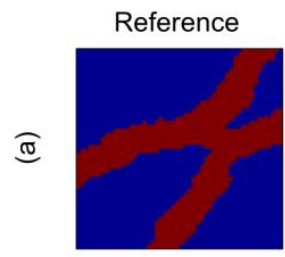

$n_{x}=n_{y}=100$
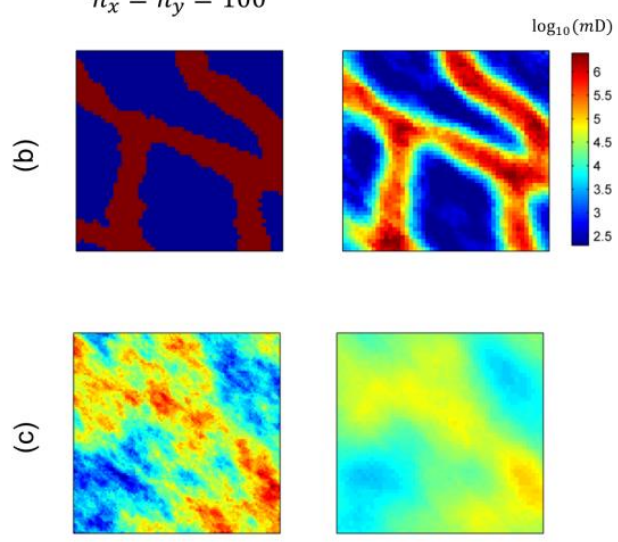

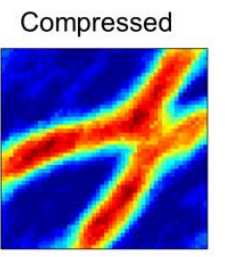

$\log _{10}(m \mathrm{D})$
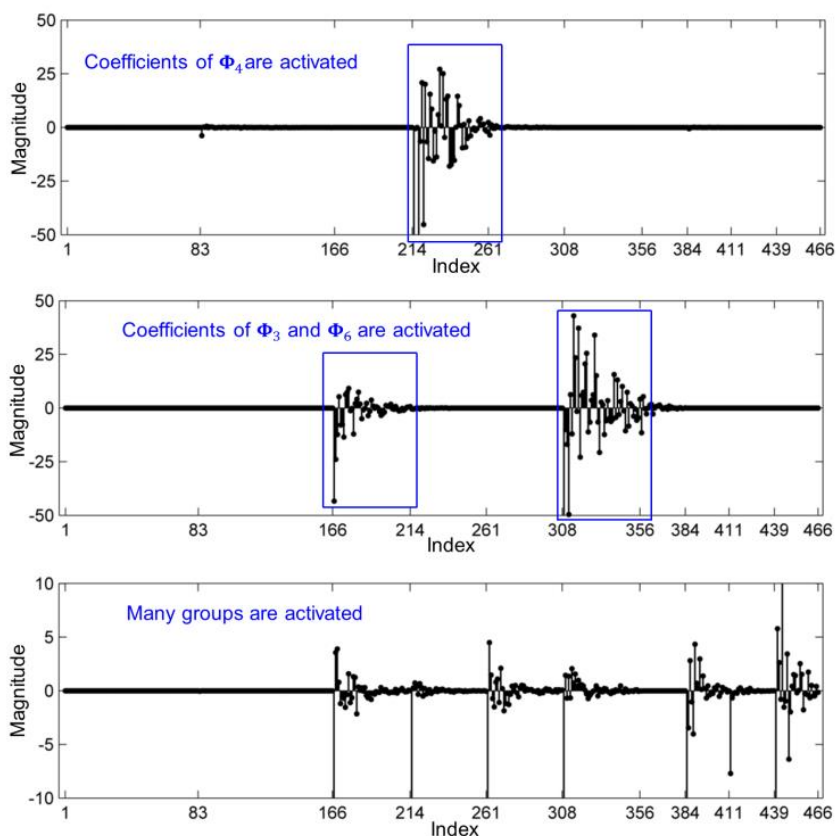

Figure 8. Best approximate representations of three models using group-sparsity regularization: (a) reference model consistent with group $\mathbf{U}_{4}$; (b) reference model has features similar to those in groups $\mathbf{U}_{3}$ and $\mathbf{U}_{6}$; (c) reference model with completely different geologic features than those in prior groups. 


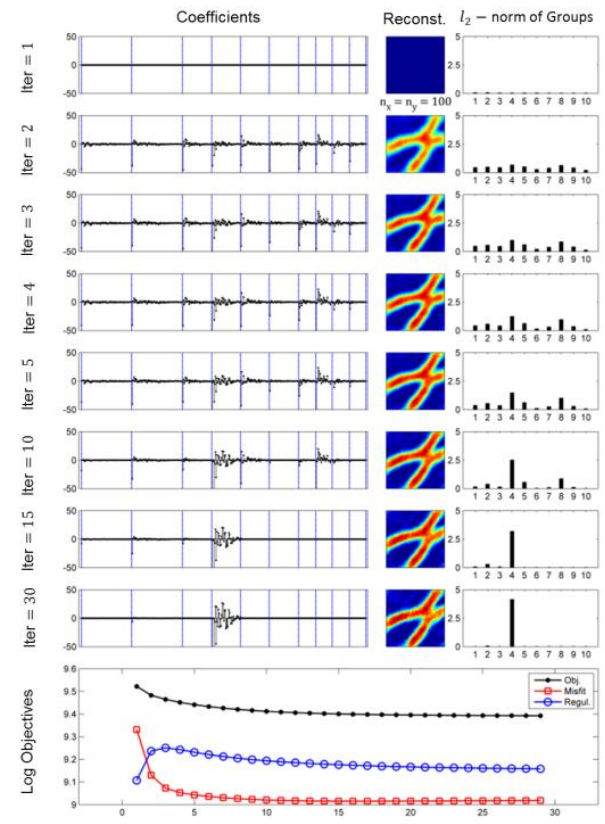

Case 1: Initial Under Estimated

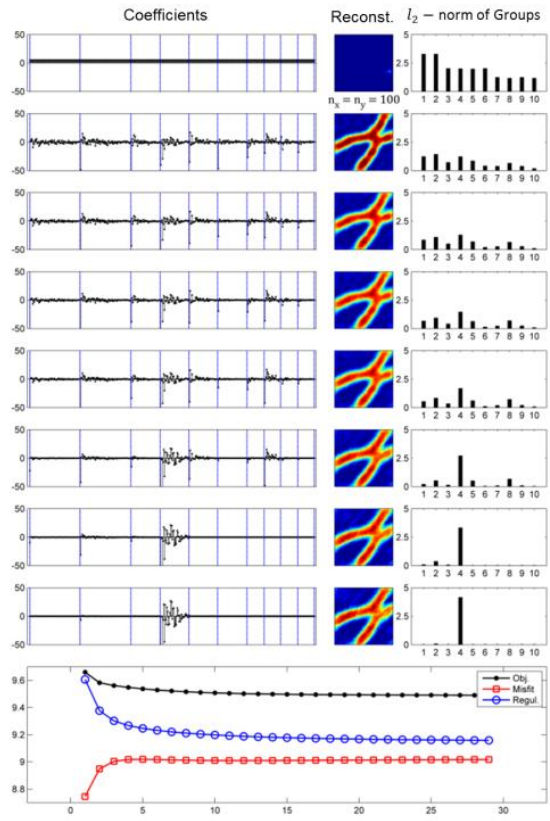

Case 2: Initial Over Estimated

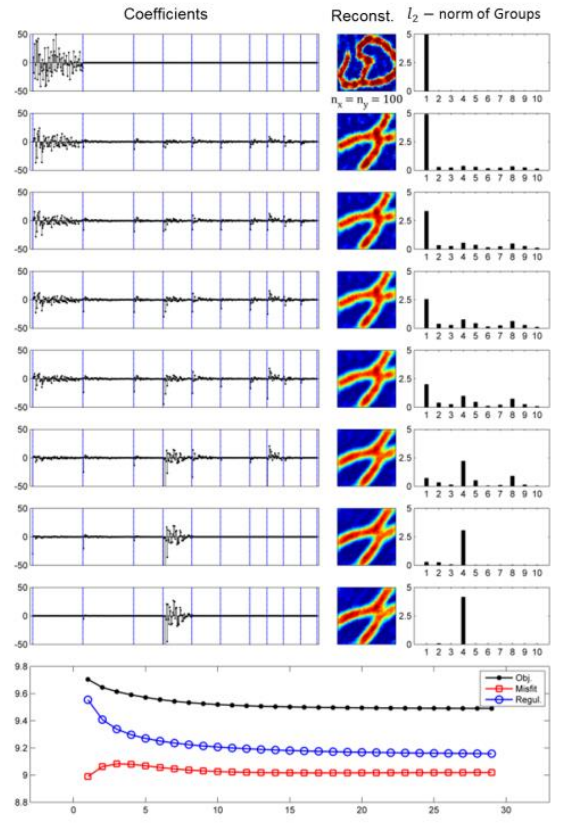

Case 3: Initial is one of the Irrelevant groups

Figure 9. The convergence behavior of group-sparsity during sample iterations, including the misfit term, the regularization terms, reconstruction coefficients and spatial maps for three different initializations: Case 1, the initial coefficients are uniformly distributed across the groups and have relatively small values; Case 2, initial coefficients are uniformly distributed and have relatively large values; Case 3, initial coefficients are zero for all groups except for an inconsistent group, for which relatively large values are assigned. 
(a) Configuration of Tomographic Inversion

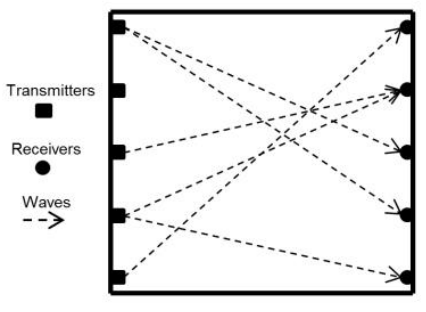

$\mathbf{U}_{1}$

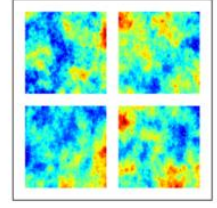

$\mathbf{U}_{3}$

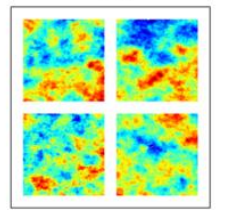

$\mathbf{U}_{2}$

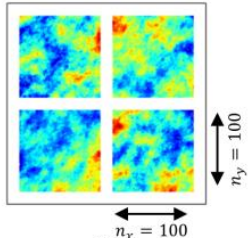

$\mathbf{U}_{4}$

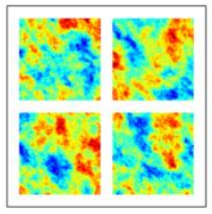

(b) Parameters of the Variogram Models

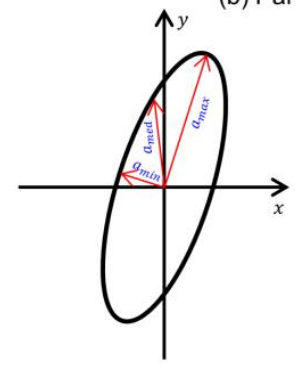

\begin{tabular}{cccc}
\hline & $\gamma_{1}$ & $\gamma_{2}$ & $\gamma_{3}$ \\
\hline$a_{\text {max }}$ & 280 & 700 & 100 \\
$a_{\text {med }}$ & 210 & 290 & 50 \\
$a_{\text {min }}$ & 140 & 100 & 50 \\
\hline \multicolumn{4}{c}{ Kriging Type : Ordinary Kriging } \\
Variogram Type : Spherical
\end{tabular}

(c) Gaussian Prior Set of Realizations

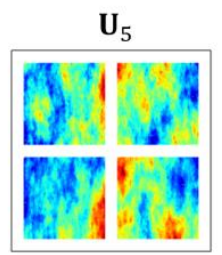

$\mathbf{U}_{7}$

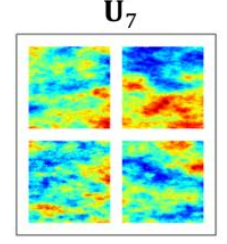

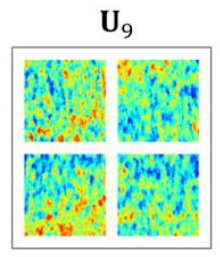

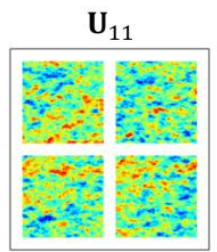

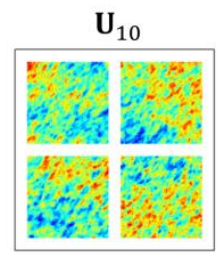

$\mathbf{U}_{12}$
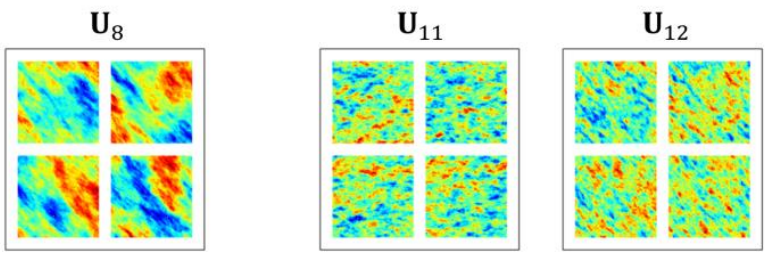

Figure 10. (a) Tomographic inversion setup; (b) Three anisotropic variogram models with specified ranges; (c) Four sample realizations (out of 500) from 12 different groups that are obtained by assigning four anisotropy directions $\theta=0^{\circ}, 45^{\circ}, 90^{\circ}, 135^{\circ}$ to each variogram model. The realizations are generated using the sgsim algorithm. 

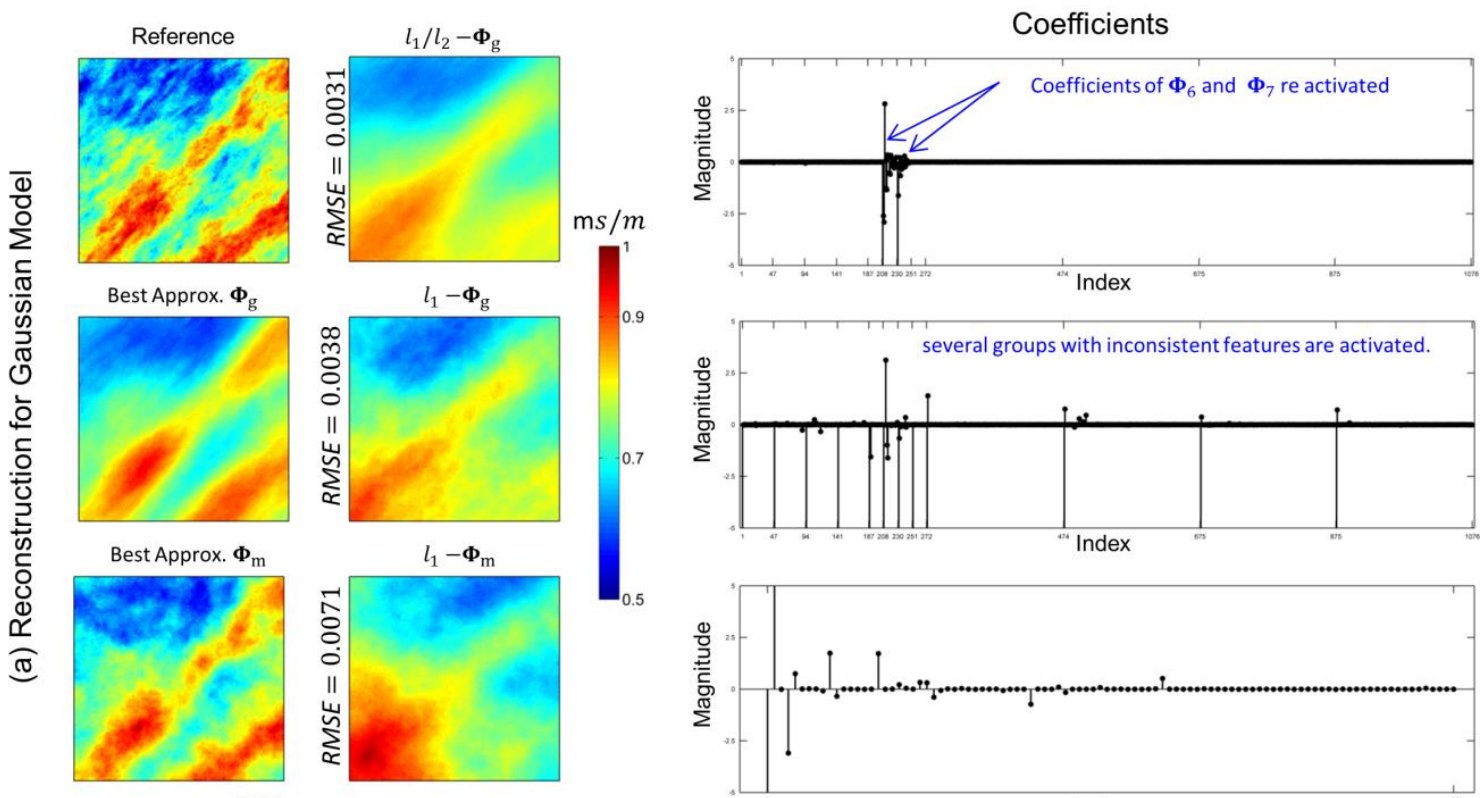

$n_{x}=n_{y}=100$
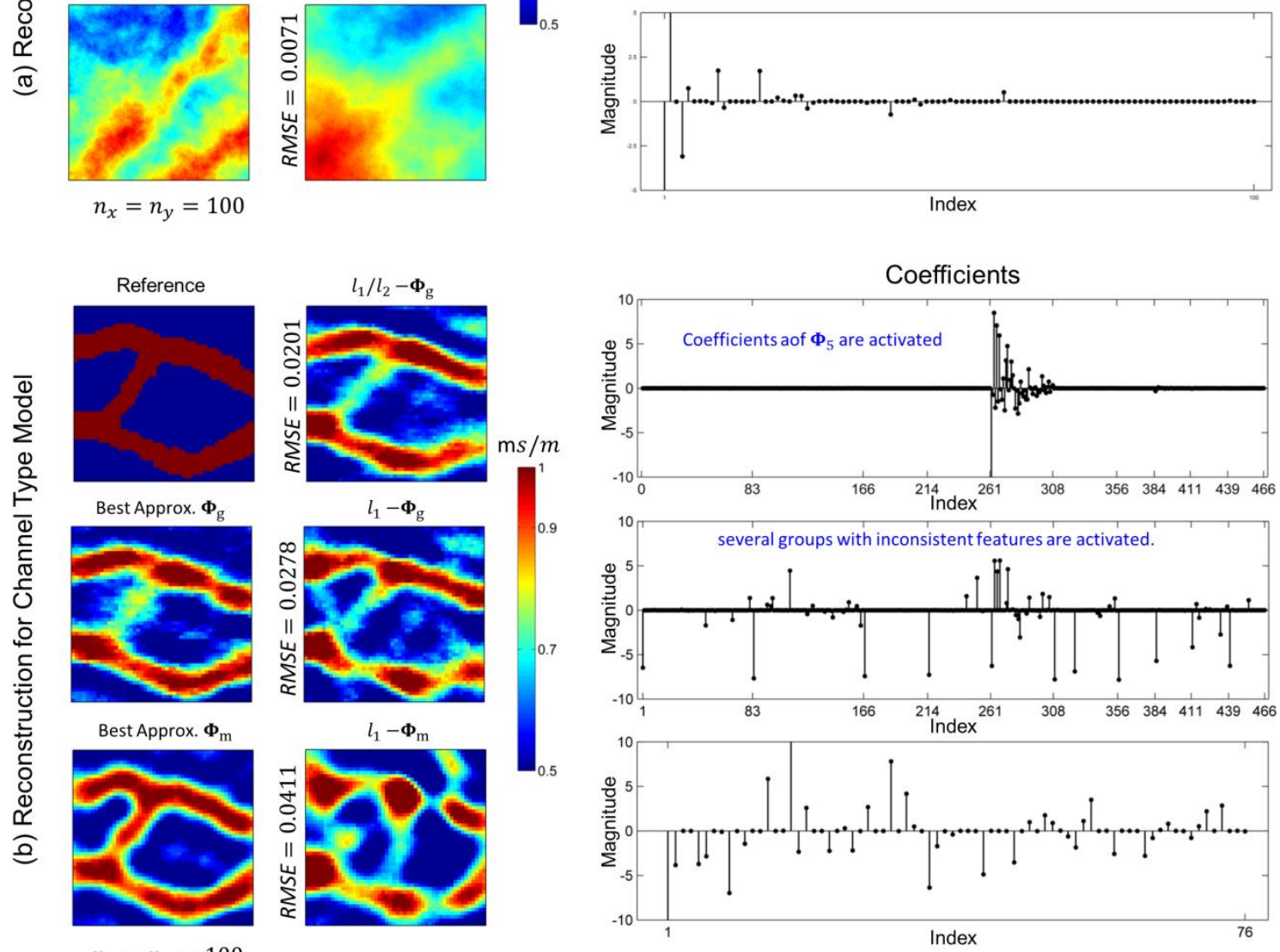

Figure 11. Tomographic inversion results for: (a) multi-Gaussian example; and (b) channel example. In each case, the figures on the left show the reference models (first row) and best approximation maps (assuming full knowledge of all grid cells) with $\boldsymbol{\Phi}_{\mathrm{g}}$ (second row) and $\boldsymbol{\Phi}_{\mathrm{m}}$ (third row) while the plots in the second column show the reconstructed maps with $l_{1} / l_{2}-$ $\boldsymbol{\Phi}_{\mathrm{g}}$ (top), $l_{1}-\boldsymbol{\Phi}_{\mathrm{g}}$ (middle) and $l_{1}-\boldsymbol{\Phi}_{\mathrm{m}}$ (bottom); the corresponding reconstructed coefficients are shown on the rightmost column. 
Complexity of the Model and Information Available from the Data
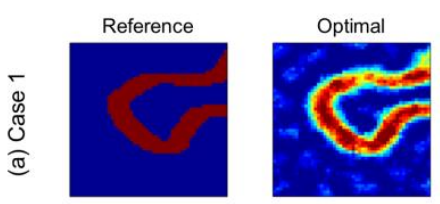

$n_{x}=n_{y}=100$

Reference

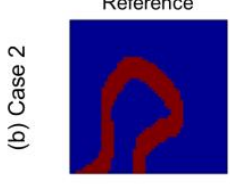

Optimal

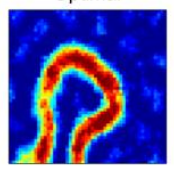

$n_{x}=n_{y}=100$

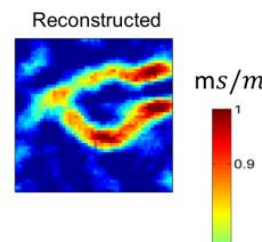

Reconstructed

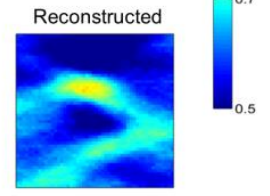

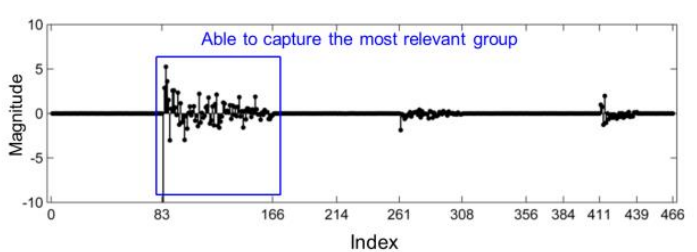

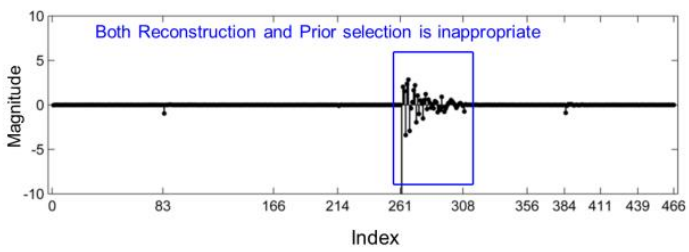

Figure 12. Effect of data content and model complexity in tomographic inversion example; two different meandering channels are used as reference slowness maps to illustrate the effect of data acquisition configuration (when two channels are intersected by the same ray path). The example in (a) shows the limitation of the TSVD basis for parameterization of complex meandering channels in ill-posed problems. 

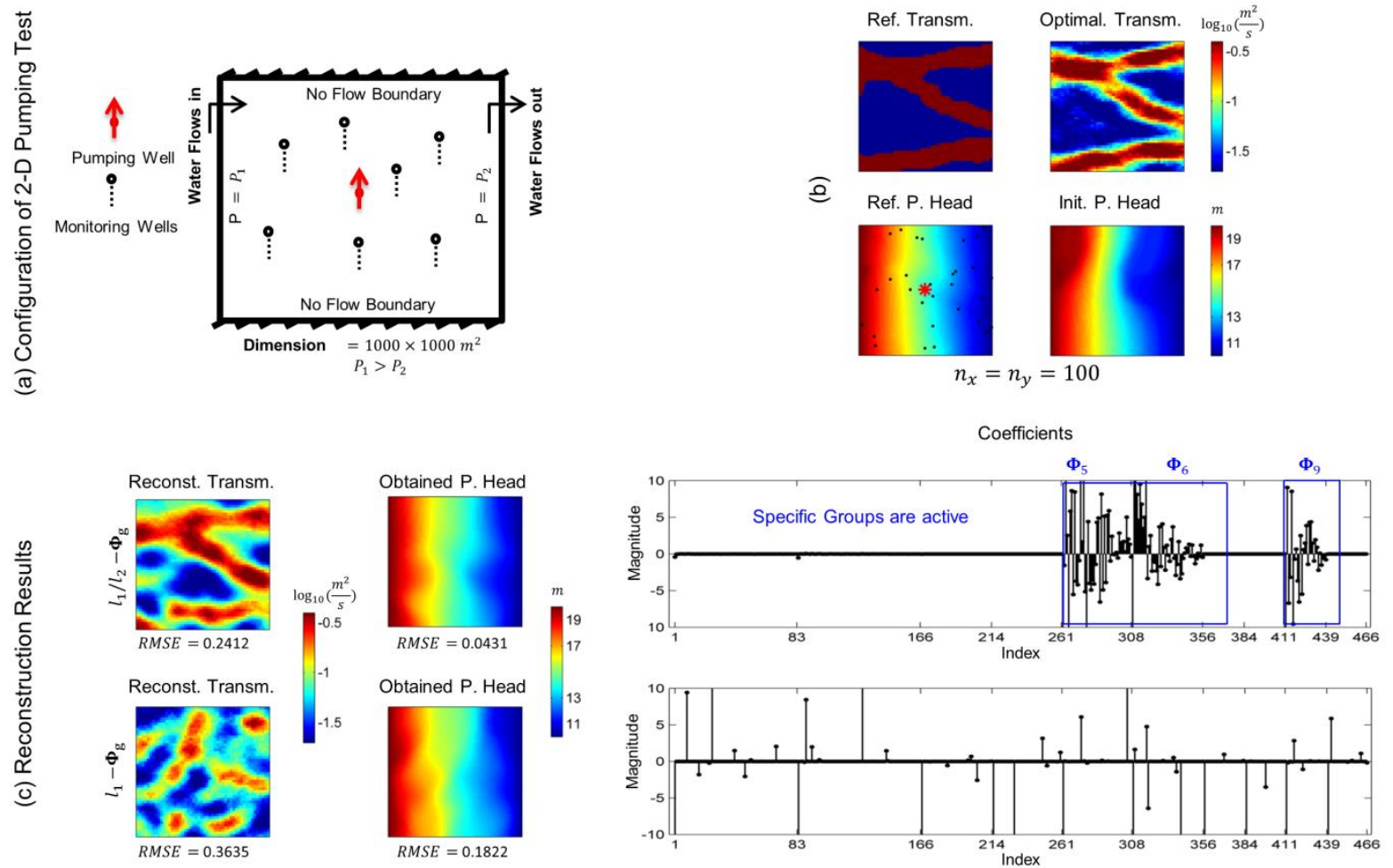

Figure 13. Model calibration results for a pumping test experiment: (a) 2D configuration of pumping test; (b) the reference transmissivity field and best achievable approximation of it (top), and the corresponding reference and initial pressure head fields (bottom); (c) transmissivity fields and pressure head after calibration using group-sparsity $\left(l_{1} / l_{2}\right.$-norm) and regular $l_{1}$-norm sparsity inducing reconstruction. 


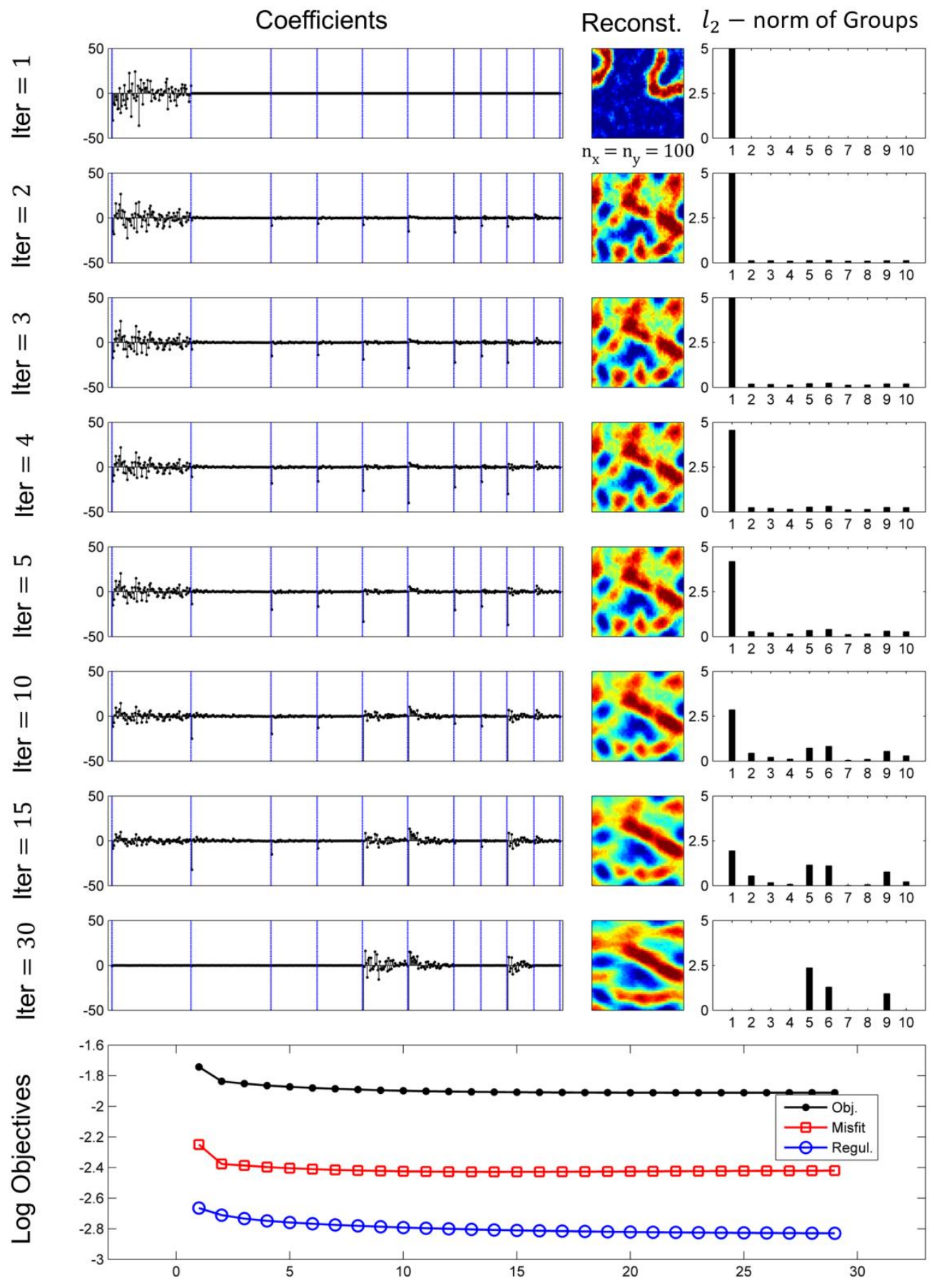

Figure 14. The behavior of the group-sparsity reconstruction during model calibration in the 2D pumping test: the first eight rows show the reconstructed coefficients (left) with their corresponding spatial maps (middle) and group contributions (left) for select iterations. The last row displays the evolution of the misfit term, regularization term, and the overall objective function. 

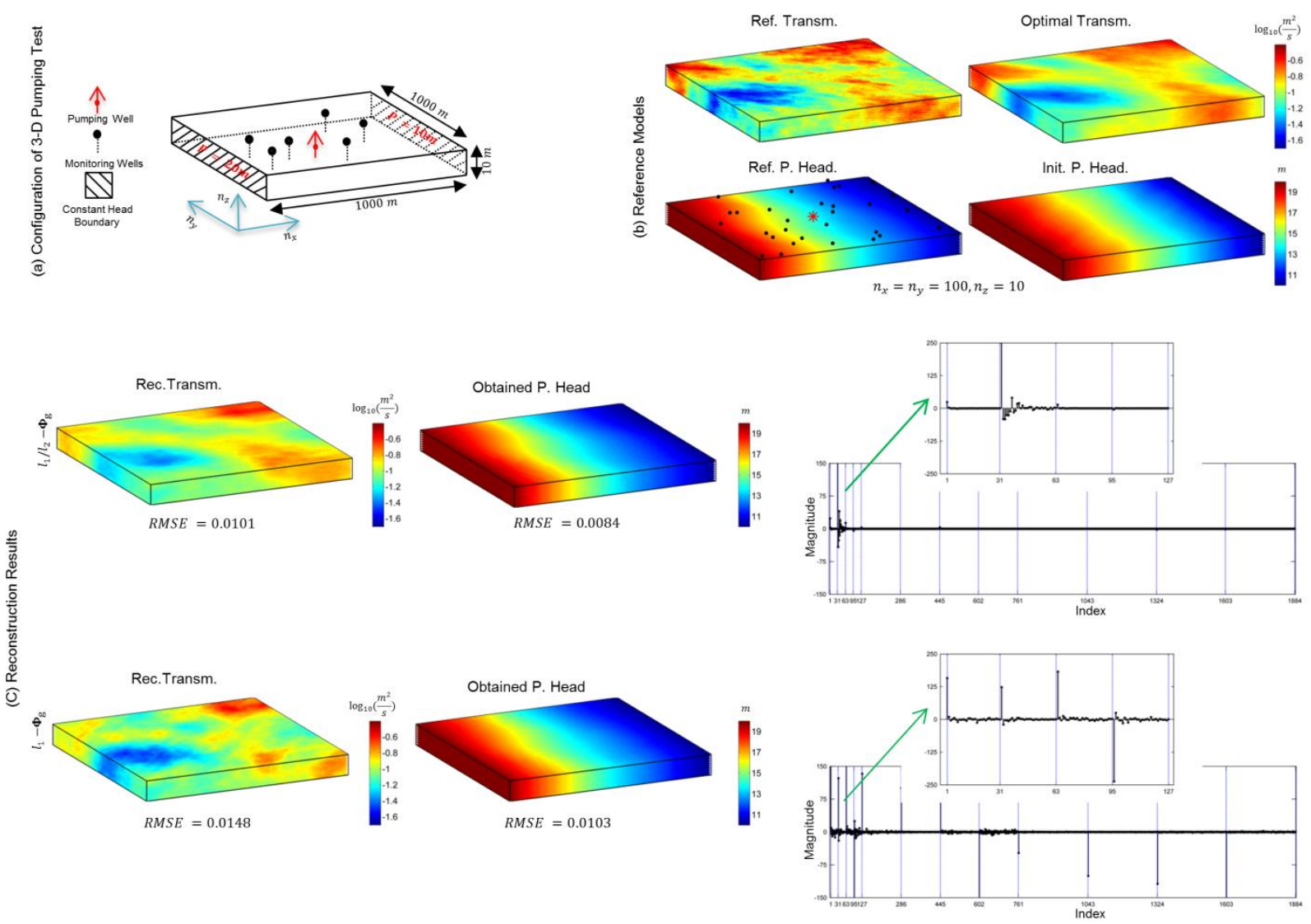

Figure 15. Reconstruction results for the 3D pumping test: (a) 3D aquifer configuration for the pumping test; (b) reference transmissivity field and the best achievable approximation (assuming perfect knowledge of the field), as well as the reference and initial pressure head fields; (c) reconstruction results for transmissivity and pressure head fields with group-sparsity $\left(l_{1} / l_{2}\right.$ norm) and regular sparsity $\left(l_{1}\right.$-norm) regularization. 

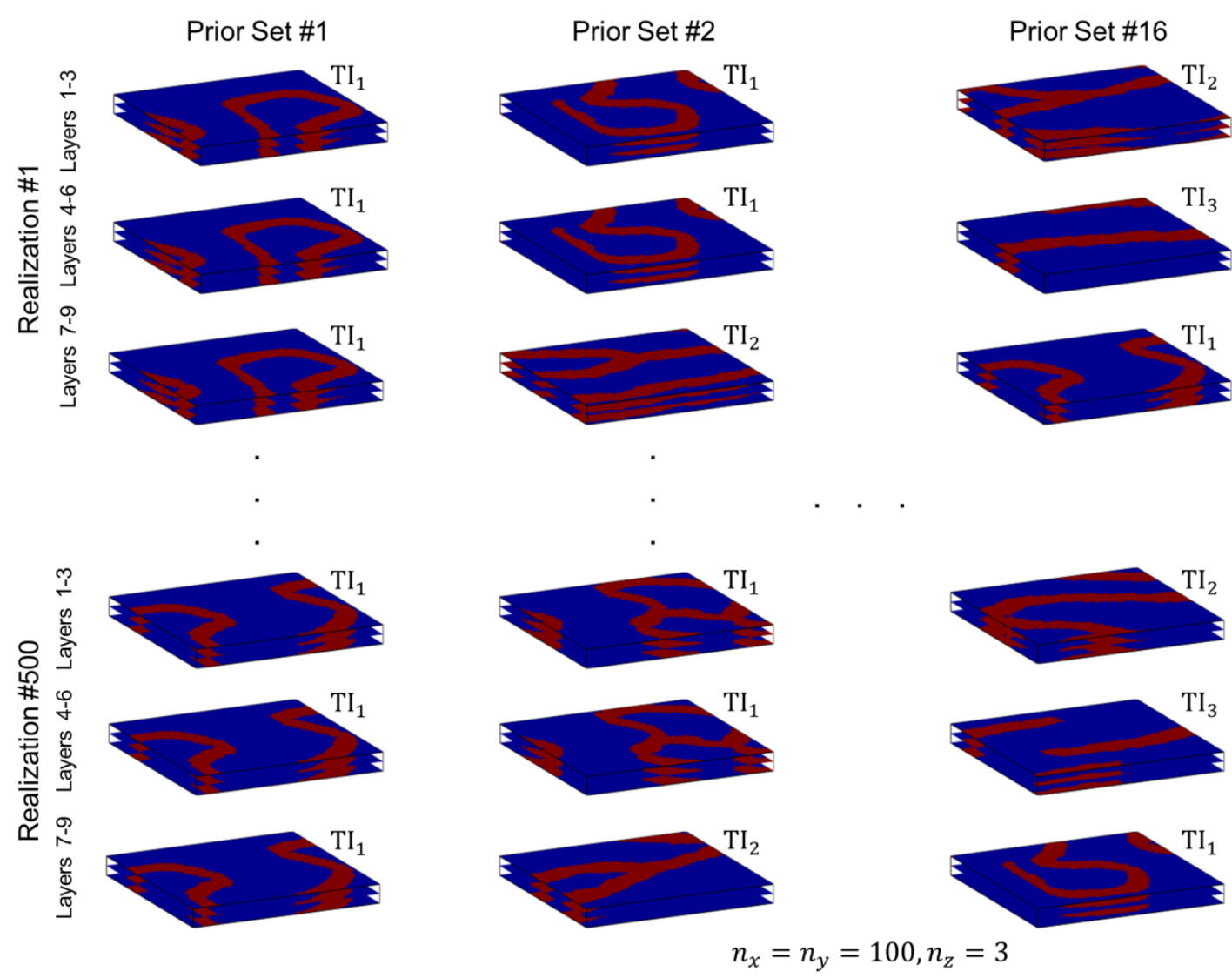

Prior Set \#27
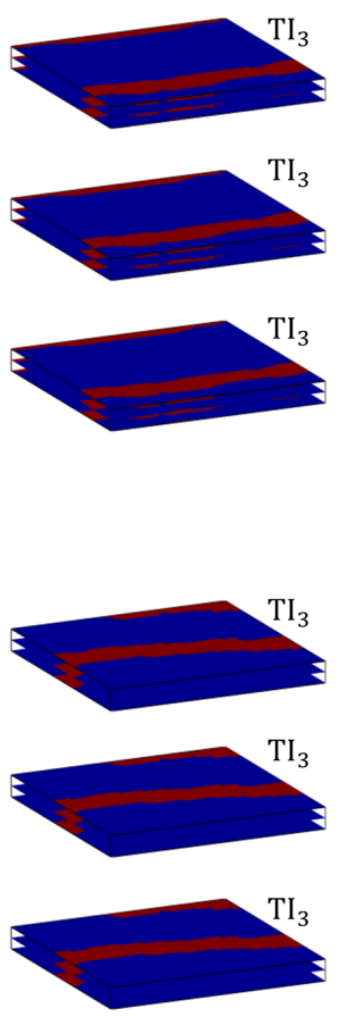

Figure 16. Two samples realizations (top and bottom) from prior scenarios 1, 2, 16, and 27 for the channel type 3D transmissivity field in Case 5. The training images $\mathrm{TI}_{1}, \mathrm{TI}_{2}$ and $\mathrm{TI}_{3}$ represent meandering, intersecting and straight channels, respectively (as depicted in Figure 5). 
(b) Detailed Reference Models in Layers

(a) Reference Models
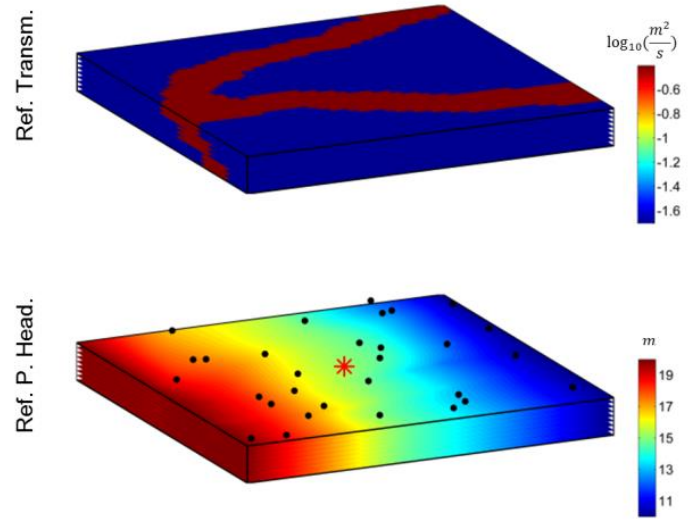

$n_{x}=n_{y}=100, n_{z}=9$

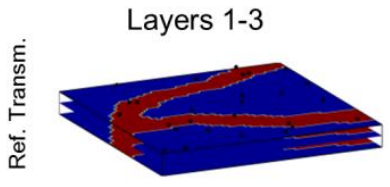

Layers 4-6
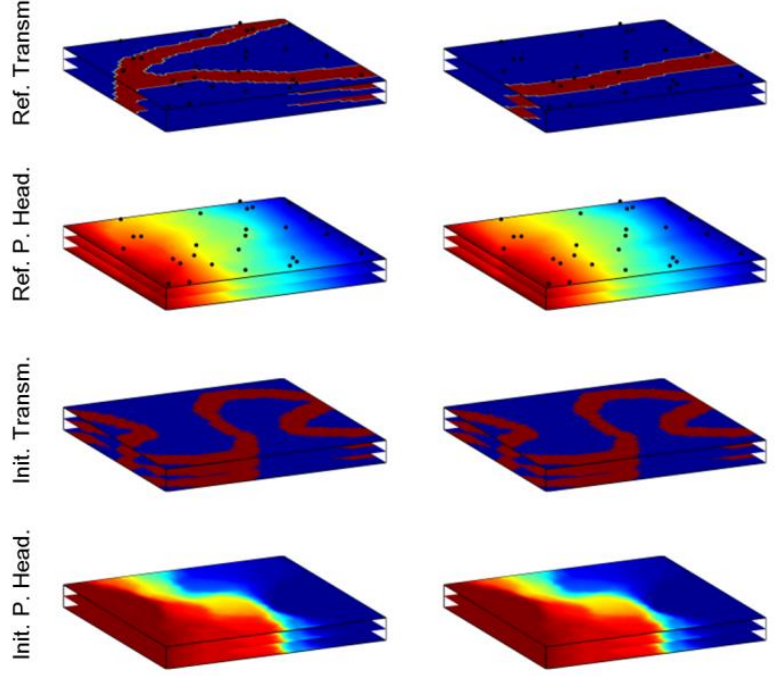

Layers 7-9
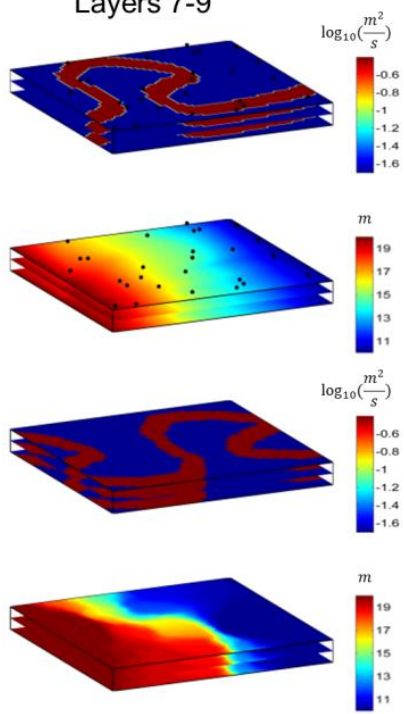

Figure 17. Reference transmissivity and pressure field for the 3D pumping test with channeltype connectivity. The locations of monitoring and pumping wells are shown with black dots and a red star, respectively. 
(a) Reconstruction Transmissivity

Layers 1-3
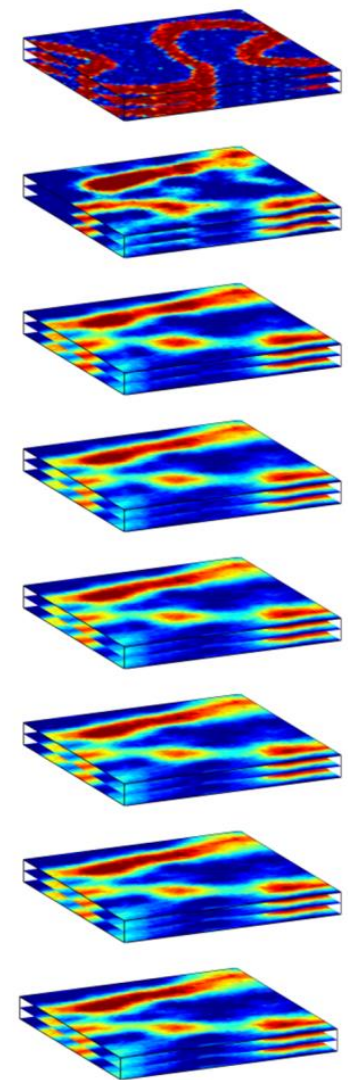

Layers 4-6
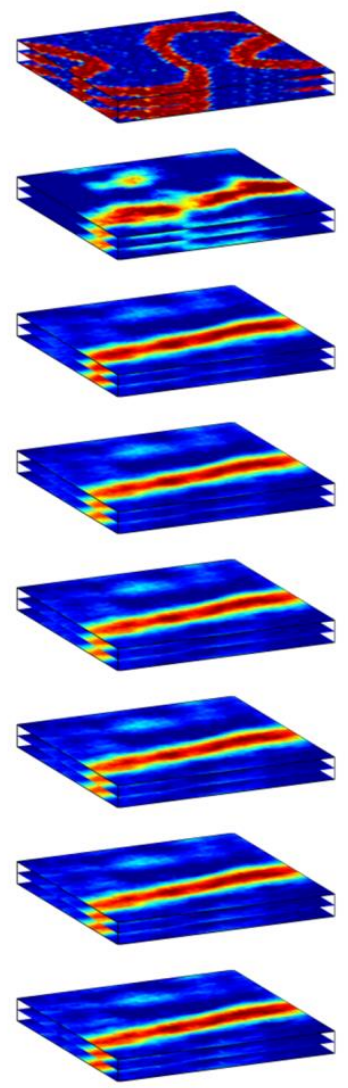

$n_{x}=n_{y}=100, n_{z}=3$
Layers 7-9
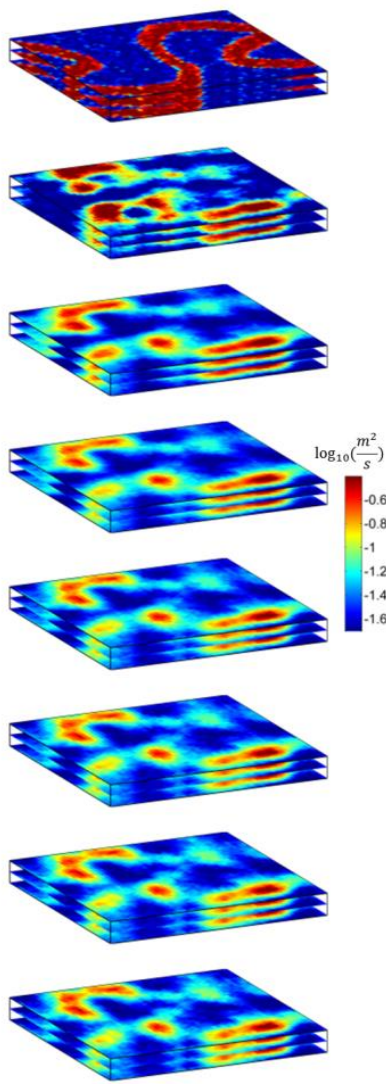

(b) $l_{2}-$ norm of the groups
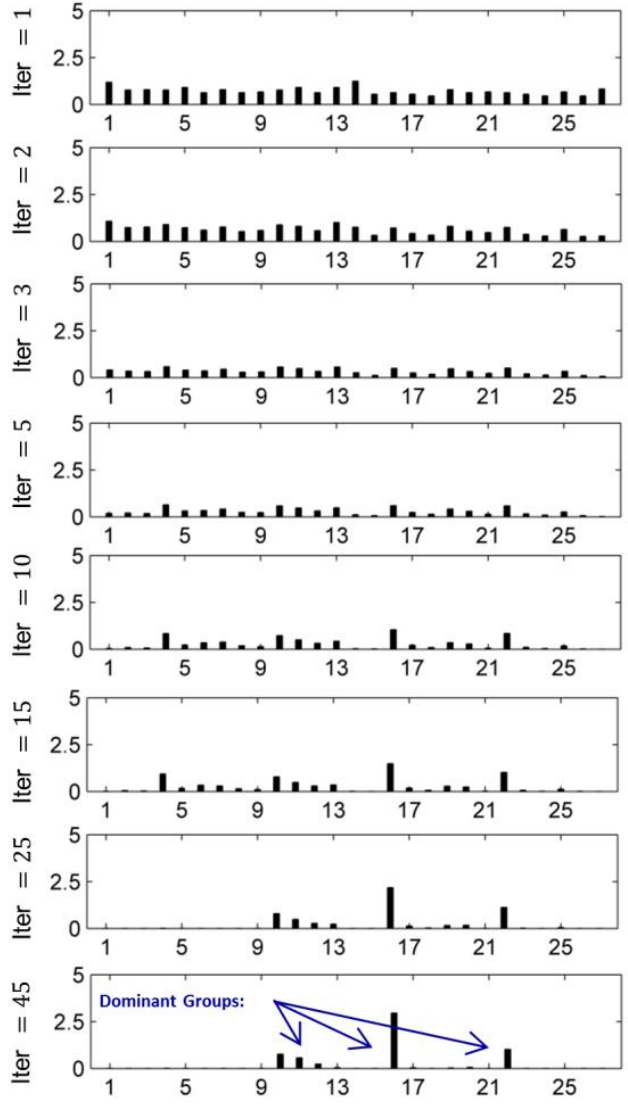

(d) Initial and Final Coefficients
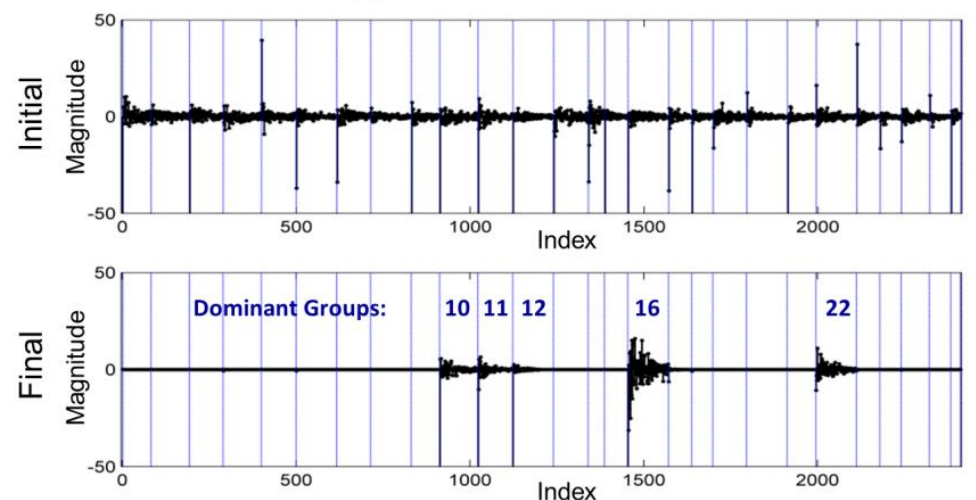

Figure 18. Reconstruction results: (a) evolution of the transmissivity field with iterations (each row shows the results for layers 1-3, 4-6 and 7-9); (b) evolution of the $l_{2}$-norm of the coefficients corresponding to the 27 prior groups (geologic scenarios) with iterations; (c): the pressure head field predicted from the final transmissivity field; (d) the initial and final active coefficients. 